\title{
LINEAR CHARACTERS OF SYLOW SUBGROUPS OF SYMMETRIC GROUPS
}

\author{
EUGENIO GIANNELLI, STACEY LAW, AND JASON LONG
}

\begin{abstract}
Let $p$ be any prime. Let $P_{n}$ be a Sylow $p$-subgroup of the symmetric group $\mathfrak{S}_{n}$. Let $\phi$ and $\psi$ be linear characters of $P_{n}$ and let $N$ be the normaliser of $P_{n}$ in $\mathfrak{S}_{n}$. In this article we show that the inductions of $\phi$ and $\psi$ to $\mathfrak{S}_{n}$ are equal if, and only if, $\phi$ and $\psi$ are $N$-conjugate. This is an analogue for symmetric groups of a result of Navarro for $p$-solvable groups.
\end{abstract}

\section{INTRODUCTION}

Let $G$ be a finite group and let $P$ be a Sylow subgroup of $G$. The algebraic properties of those characters of $G$ obtained by inducing irreducible characters of $P$ encodes interesting information about the group $G$ itself [N18]. For example, the normality of $P$ in $G$ is controlled by specific properties of the permutation character $\mathbb{1}_{P} \uparrow^{G}$. More precisely, in [MN12] it is proved that $P$ is normal in $G$ if, and only if, all the irreducible constituents of $\mathbb{1}_{P} \uparrow^{G}$ have degree coprime to $p$. On the other hand, in [NTV14] it is shown that $P$ is self-normalising if, and only if, $\mathbb{1}_{G}$ is the only constituent of $\mathbb{1}_{P} \uparrow^{G}$ having degree coprime to $p$.

Recently, the first two authors have studied the induction of characters of $P$ to $G$ in the case where $G=\mathfrak{S}_{n}$ is a symmetric group. The irreducible constituents of $\mathbb{1}_{P} \uparrow^{\mathfrak{S}_{n}}$ were completely described in GL18. Later in GL20, the focus was extended to the study of any monomial character $\phi \uparrow^{\mathfrak{S}_{n}}$, where $\phi$ is an arbitrary linear character of $P \in \operatorname{Syl}_{p}\left(\mathfrak{S}_{n}\right)$. The present article complements the study begun in GL20] by extending to symmetric groups a result proved by Navarro for $p$-solvable groups [N03, Theorem C].

Let $p$ be a prime. For any finite group $G$ and $P$ a Sylow $p$-subgroup of $G$, the normaliser $N=N_{G}(P)$ acts by conjugation on the set $\operatorname{Lin}(P)$ of linear characters of $P$. It is easy to see that if two characters are $N$-conjugate then their inductions to $N$, and hence $G$, are equal. Therefore, in order to study structural properties of such induced characters $\phi \uparrow^{G}$, it is sufficient to consider only a set of representatives for the orbits of $N$ in its action on $\operatorname{Lin}(P)$. However, is the converse true? That is, if $\phi$ and $\psi$ are two linear characters of $P$ such that $\phi \uparrow^{G}=\psi \uparrow^{G}$, must $\phi$ and $\psi$ be $N$-conjugate? As mentioned above, this was answered in the affirmative for all $p$-solvable groups by Navarro in [N03, though there exist finite groups (such as $\operatorname{PSL}(3,3)$ with $p=3$ ) for which the answer is negative. Our main result shows that the answer to this question is also affirmative for symmetric groups at all primes $p$.

Theorem A. Let $n \in \mathbb{N}$ and $p$ be any prime. Let $P_{n} \in \operatorname{Syl}_{p}\left(\mathfrak{S}_{n}\right)$ and let $N=N_{\mathfrak{S}_{n}}\left(P_{n}\right)$. Let $\phi$ and $\psi$ be linear characters of $P_{n}$. Then $\phi \uparrow_{P_{n}}^{\mathfrak{S}_{n}}=\psi \uparrow_{P_{n}}^{\mathfrak{S}_{n}}$ if, and only if, $\phi$ and $\psi$ are $N$-conjugate.

In order to prove Theorem $\underline{\mathrm{A}}$, we calculate the values of the induced characters $\phi \uparrow^{\mathfrak{S}_{n}}$ and $\psi \uparrow^{\mathfrak{S}_{n}}$ on a range of different conjugacy classes of $S_{n}$. By relating these values to combinatorial properties of $\phi$ and $\psi$, we demonstrate that if the induced characters agree on sufficiently many

2020 Mathematics Subject Classification. 20C15, 20C30. 
conjugacy classes, then in fact the linear characters $\phi$ and $\psi$ are $N$-conjugate. We believe that the closed formulae we provide in Lemma 4.4. Proposition 4.9 and Theorem 4.13 may be useful for future investigations, and could be of independent interest.

1.1. Structure of the article. We begin with a reduction of Theorem $\mathrm{A}$ to the case where $n=a p^{k}$ for some $a \in\{1,2, \ldots, p-1\}$ and $k \in \mathbb{N}$. This is stated separately as Theorem 2.2. In Section 2, we prove Theorem A assuming Theorem 2.2.

In Section 3 we describe a certain parametrisation $\phi=\phi(\underline{\mathbf{u}})$ of the linear characters of $P_{n}$ and formulate what it means for linear characters to be $N$-conjugate in terms of this parametrisation. We then state Theorem 3.6 which re-expresses Theorem 2.2 using this new notation, and whose proof will be the main aim of the remaining sections of the paper.

Section 4 represents the technical core of the argument. Here, we tackle the computation of induced character values, culminating in Theorem 4.13. While it will quickly become clear that induced character $\phi \uparrow^{\mathfrak{S}_{n}}$ can be calculated in a recursive fashion, the main difficulty will be in simplifying the resulting expressions so that they can be used to analyse the parametrisation $\phi(\underline{\mathbf{u}})$. To achieve this, we make use of a number of combinatorial tools, including identities concerning Bell polynomials and Stirling numbers of the second kind.

In Section 5 we shall use the induced character formulae from Section 4 to establish Theorem 3.6, and hence Theorem $\mathrm{A}$.

In Appendix A, we provide detailed examples to clarify some of the expressions in Section 4, The paper is self-contained and the examples are not necessary to follow the proof, but the reader may find it helpful to refer to the appendix when reading the proof of Proposition 4.9.

Acknowledgements. We thank the anonymous reviewer for his/her helpful corrections. The second author was supported by a London Mathematical Society Early Career Fellowship at the University of Oxford (ECF-1819-05).

\section{REDUCTION TO $n=a p^{k}$}

We begin by introducing some notation used throughout the article. We refer the reader to [JK81] for a detailed account of the representation theory of symmetric groups.

For a finite group $G$, let $\operatorname{Char}(G)$ denote the set of ordinary characters of $G$. Let $\operatorname{Irr}(G)$ the subset of those which are irreducible, and $\operatorname{Lin}(G)$ for those which are linear, i.e. of degree 1 . For $m$ a natural number, let $[m]$ denote the set $\{1,2, \ldots, m\}$. The symmetric group on the set $[n]=\{1,2, \ldots, n\}$ will be denoted by $\mathfrak{S}_{n}$ throughout.

First, we record a proof of the easy direction of Theorem A.

Lemma 2.1. Let $G$ be a finite group and $p$ be a prime. Let $P \in \operatorname{Syl}_{p}(G)$ and $N=N_{G}(P)$. Suppose $\phi, \psi \in \operatorname{Char}(P)$ and $\psi=\phi^{m}$ for some $m \in N$. Then $\phi \uparrow_{P}^{G}=\psi \uparrow_{P}^{G}$.

Proof. Let $\alpha \in \operatorname{Irr}(N)$. Note that $\alpha^{m}=\alpha$. Then by Frobenius reciprocity,

$$
\left\langle\phi \uparrow^{N}, \alpha\right\rangle=\left\langle\phi, \alpha \downarrow_{P}\right\rangle=\left\langle\phi^{m},\left(\alpha \downarrow_{P}\right)^{m}\right\rangle=\left\langle\psi,\left(\alpha^{m}\right) \downarrow_{P}\right\rangle=\left\langle\psi \uparrow^{N}, \alpha\right\rangle .
$$

Thus $\phi \uparrow^{N}=\psi \uparrow^{N}$, as $\alpha$ is arbitrary. It follows that $\phi \uparrow_{P}^{G}=\psi \uparrow_{P}^{G}$.

The difficult part of Theorem $\mathrm{A}$ is the converse of Lemma 2.1. It turns out that this converse reduces to the case when $n=a p^{k}$, which we now state.

Theorem 2.2. Let $a \in[p-1]$ and $k \in \mathbb{N}$. Let $\phi, \psi \in \operatorname{Lin}\left(P_{a p^{k}}\right)$ be such that $\phi \uparrow^{\mathfrak{S}_{a p^{k}}}=\psi \uparrow^{\mathfrak{S}_{a p^{k}}}$. Then $\phi$ and $\psi$ are $N_{\mathfrak{S}_{a p^{k}}}\left(P_{a p^{k}}\right)$-conjugate. 
Theorem 2.2 is proved in Section 5 , following explicit calculations of certain induced character values in Section 4. In the rest of this section, we deduce Theorem A from Theorem 2.2 .

Recall that for each $n \in \mathbb{N}$, $\operatorname{Irr}\left(\mathfrak{S}_{n}\right)$ is naturally in bijection with the set of all partitions of $n$. For a partition $\lambda$ of the number $n$, also written $\lambda \vdash n$, we denote the corresponding irreducible character of $\mathfrak{S}_{n}$ by $\chi^{\lambda}$.

Lemma 2.3. Let $n, m \in \mathbb{N}$. Let $A, B \in \operatorname{Char}\left(\mathfrak{S}_{n}\right)$ and $Z \in \operatorname{Char}\left(\mathfrak{S}_{m}\right)$. Then

$$
(A \times Z) \uparrow_{\mathfrak{S}_{n} \times \mathfrak{S}_{m}}^{\mathfrak{S}_{n+m}}=(B \times Z) \uparrow \underset{\mathfrak{S}_{n} \times \mathfrak{S}_{m}}{\mathfrak{S}_{n+m}} \text { if and only if } A=B
$$

Proof. The 'if' direction is clear, so now suppose that $(A \times Z) \uparrow^{\mathfrak{S}_{n+m}}=(B \times Z) \uparrow^{\mathfrak{S}_{n+m}}$ and assume for a contradiction that $A \neq B$. For $X \in\{A, B, Z\}$, we let $c_{\lambda}^{X}:=\left\langle X, \chi^{\lambda}\right\rangle$, where $\lambda$ is a partition of $n$ (respectively $m$ ) if $X \in\{A, B\}$ (respectively $X=Z$ ). We define the following sets:

$$
\mathcal{M}=\left\{\lambda \vdash n \mid c_{\lambda}^{A} \neq c_{\lambda}^{B}\right\} \text { and } \mathcal{N}=\left\{\mu \vdash m \mid c_{\mu}^{Z} \neq 0\right\},
$$

which by assumption are non-empty. Let $\bar{\lambda}$ and $\bar{\mu}$ be the lexicographically greatest partitions in $\mathcal{M}$ and $\mathcal{N}$ respectively, and let $\alpha$ be the partition of $n+m$ defined by $\alpha=\bar{\lambda}+\bar{\mu}:=$ $\left(\lambda_{1}+\mu_{1}, \lambda_{2}+\mu_{2}, \ldots\right)$. By the Littlewood-Richardson rule [J78, Chapter 16], we have that

$$
\begin{aligned}
\left\langle(A \times Z) \uparrow \mathfrak{S}_{n+m}, \chi^{\alpha}\right\rangle & =c_{\bar{\lambda}}^{A} c_{\bar{\mu}}^{Z} c_{\bar{\lambda} \bar{\mu}}^{\alpha}+\sum_{\lambda>\bar{\lambda}} \sum_{\mu \in \mathcal{N}} c_{\lambda}^{A} c_{\mu}^{Z} c_{\lambda \mu}^{\alpha}=c_{\bar{\lambda}}^{A} c_{\bar{\mu}}^{Z} c_{\bar{\lambda} \bar{\mu}}^{\alpha}+\sum_{\lambda>\bar{\lambda}} \sum_{\mu \in \mathcal{N}} c_{\lambda}^{B} c_{\mu}^{Z} c_{\lambda \mu}^{\alpha} \\
& \neq c c_{\bar{\lambda}} c_{\frac{Z}{\mu}} c_{\bar{\lambda} \bar{\mu}}^{\alpha}+\sum_{\lambda>\bar{\lambda}} \sum_{\mu \in \mathcal{N}} c_{\lambda}^{B} c_{\mu}^{Z} c_{\lambda \mu}^{\alpha}=\left\langle(B \times Z) \uparrow \mathfrak{S}_{n+m}, \chi^{\alpha}\right\rangle .
\end{aligned}
$$

This is a contradiction, and the proof is concluded.

Lemma 2.4. Let $b, n, m \in \mathbb{N}$ with $n>m$. Let $P \times Q \leq \mathfrak{S}_{b n} \times \mathfrak{S}_{m} \leq \mathfrak{S}_{b n+m}$ be such that $P$ contains an element $\sigma$ which is a product of $b$ disjoint $n$-cycles. Let $g \in Q$. Let $\chi$ and $\eta$ be class functions of $P$ and $Q$ respectively. Then

$$
(\chi \times \eta) \uparrow_{P \times Q}^{\mathfrak{S}_{b n+m}}(\sigma g)=\chi \uparrow_{P}^{\mathfrak{S}_{b n}}(\sigma) \cdot \eta \uparrow_{Q}^{\mathfrak{S}_{m}}(g) .
$$

Proof. This follows from the definition of induction, after observing that $\sigma^{x} \in P \times Q$ if and only if $\sigma^{x} \in P$, for all $x \in \mathfrak{S}_{b n+m}$.

Assuming Theorem 2.2, we now prove Theorem A.

Proof of Theorem A. Recall that Lemma 2.1 gives the easy direction of Theorem A. We now prove the difficult direction. Let $n=a_{1} p^{n_{1}}+\cdots+a_{t} p^{n_{t}}$ be the $p$-adic expansion of $n$, with $t \in \mathbb{N}$, $a_{i} \in[p-1]$ for all $i$ and $0 \leq n_{1}<\cdots<n_{t}$. Suppose $\phi \uparrow_{P_{n}}^{\mathfrak{S}_{n}}=\psi \uparrow_{P_{n}}^{\mathfrak{S}_{n}}$. We wish to show that $\phi$ and $\psi$ are $N_{\mathfrak{S}_{n}}\left(P_{n}\right)$-conjugate.

We proceed by induction on $t$, noting that Theorem 2.2 gives the case of $t=1$. Now suppose $t \geq 2$ and assume for a contradiction that $\phi$ and $\psi$ are not $N_{\mathfrak{S}_{n}}\left(P_{n}\right)$-conjugate. Let $m=a_{t} p^{n_{t}}$ and write $\phi=\phi_{1} \times \phi_{2}$ and $\psi=\psi_{1} \times \psi_{2}$ where $\phi_{1}, \psi_{1} \in \operatorname{Lin}\left(P_{m}\right)$ and $\phi_{2}, \psi_{2} \in \operatorname{Lin}\left(P_{n-m}\right)$. Since $\phi$ and $\psi$ are not $N_{\mathfrak{S}_{n}}\left(P_{n}\right)$-conjugate and $N_{\mathfrak{S}_{n}}\left(P_{n}\right) \cong N_{\mathfrak{S}_{m}}\left(P_{m}\right) \times N_{\mathfrak{S}_{n-m}}\left(P_{n-m}\right)$ (see [L19, §2.3.2], for instance), at least one of the following two statements must hold:

(i) $\phi_{1}$ and $\psi_{1}$ are not $N_{\mathfrak{S}_{m}}\left(P_{m}\right)$-conjugate;

(ii) $\phi_{2}$ and $\psi_{2}$ are not $N_{\mathfrak{S}_{n-m}}\left(P_{n-m}\right)$-conjugate.

Since $P_{n} \cong P_{m} \times P_{n-m}$, we have that

$$
\left(\phi_{1} \uparrow_{P_{m}}^{\mathfrak{S}_{m}} \times \phi_{2} \uparrow_{P_{n-m}}^{\mathfrak{S}_{n-m}}\right) \uparrow^{\mathfrak{S}_{n}}=\phi \uparrow_{P_{n}}^{\mathfrak{S}_{n}}=\psi \uparrow_{P_{n}}^{\mathfrak{S}_{n}}=\left(\psi_{1} \uparrow_{P_{m}}^{\mathfrak{S}_{m}} \times \psi_{2} \uparrow_{P_{n-m}}^{\mathfrak{S}_{n-m}}\right) \uparrow^{\mathfrak{S}_{n}}
$$


and so using Lemmas 2.1, 2.3 and the inductive hypothesis, we deduce that both conditions (i) and (ii) must hold. Let $g \in \mathfrak{S}_{n-m}$ be such that $\phi_{2} \uparrow^{\mathfrak{S}_{n-m}}(g) \neq \psi_{2} \uparrow^{\mathfrak{S}_{n-m}}(g)$; such an element exists by the inductive hypothesis. Let $\sigma \in P_{m} \leq \mathfrak{S}_{m}$ be a product of $a_{t}$ disjoint $p^{n_{t}}$-cycles. We now denote by $h$ the element of $\mathfrak{S}_{m} \times \mathfrak{S}_{n-m} \leq \mathfrak{S}_{n}$ defined as follows:

$$
h= \begin{cases}\sigma & \text { if } \phi_{1} \uparrow^{\mathfrak{S}_{m}}(\sigma) \neq \psi_{1} \uparrow^{\mathfrak{S}_{m}}(\sigma), \\ \sigma g & \text { otherwise. }\end{cases}
$$

We remark that $\phi \uparrow^{\mathfrak{S}_{m}}(\sigma) \neq 0$ for all $\phi \in \operatorname{Lin}\left(P_{m}\right)$ : this follows from Corollary 4.5 below. (The proof could be included here, but it will be much shorter to describe following the introduction of relevant notation and ideas later.) Then $\phi \uparrow^{\mathfrak{S}_{n}}(h) \neq \psi \uparrow^{\mathfrak{S}_{n}}(h)$ by Lemma2.4, a contradiction.

\section{Wreath PROduCts AND The Structure of the Sylow SUbGroup}

We recall some basic facts concerning wreath products and the representation theory of the Sylow subgroups $P_{n}$ of $\mathfrak{S}_{n}$ (see [JK81, Chapter 4] for further detail). In Section 3.2 we describe a parametrisation of the linear characters of $P_{n}$ which will play a central role in our calculations of induced character values.

3.1. Wreath products. Let $G$ be a finite group, $n \in \mathbb{N}$ and $H \leq \mathfrak{S}_{n}$. We denote by $G^{\times n}$ the direct product of $n$ copies of $G$. The natural action of $\mathfrak{S}_{n}$ on the direct factors of $G^{\times n}$ induces an action of $\mathfrak{S}_{n}$ (and therefore of $H$ ) via automorphisms of $G^{\times n}$, giving the wreath product $G \imath H:=G^{\times n} \rtimes H$. As in [JK81, Chapter 4], we denote the elements of $G \imath H$ by $\left(g_{1}, \ldots, g_{n} ; h\right)$ for $g_{i} \in G$ and $h \in H$. Let $V$ be a $\mathbb{C} G$-module and suppose it affords the character $\phi$. We let $V^{\otimes n}:=V \otimes \cdots \otimes V$ be the corresponding $\mathbb{C} G^{\times n}$-module. The left action of $G$ 々 $H$ on $V^{\otimes n}$ defined by linearly extending

$$
\left(g_{1}, \ldots, g_{n} ; h\right): \quad v_{1} \otimes \cdots \otimes v_{n} \longmapsto g_{1} v_{h^{-1}(1)} \otimes \cdots \otimes g_{n} v_{h^{-1}(n)}
$$

turns $V^{\otimes n}$ into a $\mathbb{C}(G \succ H)$-module, which we denote by $\widetilde{V^{\otimes n}}$ (see [JK81, (4.3.7)]). We denote by $\tilde{\phi}$ the character afforded by the $\mathbb{C}(G \succ H)$-module $\widehat{V^{\otimes n}}$. For any ordinary character $\psi$ of $H$, we let $\psi$ also denote its inflation to $G$ 々 $H$ and let

$$
\mathcal{X}(\phi ; \psi):=\tilde{\phi} \cdot \psi
$$

be the ordinary character of $G \imath H$ obtained as the product of $\tilde{\phi}$ and $\psi$.

We record some basic results that will be useful later.

Lemma 3.1 ([JK81, Lemma 4.3.9]). Let $n \in \mathbb{N}$. Let $H \leq \mathfrak{S}_{n}$ and $G$ be finite groups. Let $\phi \in \operatorname{Irr}(G)$ and $\psi \in \operatorname{Irr}(H)$. Then for all $f_{1}, \ldots, f_{n} \in G$ and $\pi \in H$,

$$
\mathcal{X}(\phi ; \psi)\left(f_{1}, \ldots, f_{n} ; \pi\right)=\prod_{v=1}^{c(\pi)} \phi\left(f_{j_{v}} \cdot f_{\pi^{-1}\left(j_{v}\right)} \cdot f_{\pi^{-2}\left(j_{v}\right)} \cdots f_{\pi^{-l_{v}+1}\left(j_{v}\right)}\right) \cdot \psi(\pi),
$$

where $c(\pi)$ is the number of disjoint cycles in $\pi, l_{v}$ is the length of the $v^{\text {th }}$ cycle, and for each $v, j_{v}$ is some fixed element in the $v^{\text {th }}$ cycle.

For example, if $n=8$ and $\pi=(1,3,7,2)(5,8,6)(4)$, then $\mathcal{X}(\phi ; \psi)\left(f_{1}, \ldots, f_{8} ; \pi\right)=\phi\left(f_{2} f_{7} f_{3} f_{1}\right)$. $\phi\left(f_{6} f_{8} f_{5}\right) \cdot \phi\left(f_{4}\right) \cdot \psi(\pi)$.

Lemma 3.2. [L19, Lemma 2.22] Let $k \in \mathbb{N}$ and $p$ be a prime. Let $x=\left(f_{1}, \ldots, f_{p} ; \sigma\right) \in P_{p^{k}}$ for some $f_{i} \in P_{p^{k-1}}$ and $\sigma \in P_{p}$. If $x$ has a fixed point, then $\sigma=1$. Moreover, $x$ is a $p^{k}$-cycle if and only if $\sigma \neq 1$ and $f_{\sigma^{p-1}(1)} \cdots f_{\sigma(1)} \cdot f_{1}$ is a $p^{k-1}$-cycle. 
3.2. The representation theory of the Sylow subgroups of $\mathfrak{S}_{n}$. Fix a prime $p$ and let $n \in \mathbb{N}$. As in [L19, §2.3.2], for every $n$ we fix a Sylow $p$-subgroup $P_{n}$ of $\mathfrak{S}_{n}$ such that $P_{m} \leq P_{n}$ whenever $m$ is a $p$-adic subsum of $n$. Clearly $P_{1}$ is trivial while $P_{p}$ is cyclic of order $p$. More generally, $P_{p^{k}}=\left(P_{p^{k-1}}\right)^{\times p} \rtimes P_{p}=P_{p^{k-1}} \curlywedge P_{p} \cong P_{p} \prec \cdots \curlywedge P_{p}$ ( $k$-fold wreath product) for all $k \in \mathbb{N}$. For an arbitrary natural number $n$, suppose it has $p$-adic expansion $n=\sum_{i=1}^{t} a_{i} p^{n_{i}}$. That is, $t \in \mathbb{N}, 0 \leq n_{1}<\cdots<n_{t}$ and $a_{i} \in[p-1]$ for all $i$. Then $P_{n} \cong\left(P_{p^{n_{1}}}\right)^{\times a_{1}} \times \cdots \times\left(P_{p^{n_{t}}}\right)^{\times a_{t}}$.

We fix a parametrisation of the linear characters of $P_{n}$ for all $n$ as follows (see [L19, §2.3.2] or [GL20] for more detail). First we consider the case $n=p^{k}$. When $k=1$, observe that $\operatorname{Lin}\left(P_{p}\right)=\operatorname{Irr}\left(P_{p}\right)$. When $k \geq 2$, [I76, Corollary 6.17] gives

$$
\operatorname{Lin}\left(P_{p^{k}}\right)=\left\{\mathcal{X}(\phi ; \psi): \phi \in \operatorname{Lin}\left(P_{p^{k-1}}\right), \psi \in \operatorname{Lin}\left(P_{p}\right)\right\} .
$$

This gives a natural bijection 1 between $\operatorname{Lin}\left(P_{p^{k}}\right)$ and $\left(\operatorname{Lin}\left(P_{p}\right)\right)^{k}$. More precisely, we may parametrise $\operatorname{Lin}\left(P_{p^{k}}\right)$ by writing

$$
\operatorname{Lin}\left(P_{p^{k}}\right)=\left\{\mathcal{X}\left(\phi_{1}, \ldots, \phi_{k}\right): \phi_{i} \in \operatorname{Lin}\left(P_{p}\right)\right\}
$$

where $\mathcal{X}\left(\phi_{1}, \ldots, \phi_{k}\right)$ is defined recursively via $\mathcal{X}\left(\phi_{1}\right)=\phi_{1}$ when $k=1$, and

$$
\mathcal{X}\left(\phi_{1}, \ldots, \phi_{k}\right):=\mathcal{X}\left(\mathcal{X}\left(\phi_{1}, \ldots, \phi_{k-1}\right) ; \phi_{k}\right)
$$

when $k \geq 2$. The following lemma characterises when two linear characters of $P_{p^{k}}$ are conjugate via an element of $N_{\mathfrak{S}_{p^{k}}}\left(P_{p^{k}}\right)$ in terms of this parametrisation. Here $\mathbb{1}_{P_{p}}$ denotes the trivial character of $P_{p}$.

Lemma 3.3. Let $k \in \mathbb{N}$ and let $\theta_{1}, \theta_{2} \in \operatorname{Lin}\left(P_{p^{k}}\right)$. Suppose $\theta_{1}=\mathcal{X}\left(\phi_{1}, \ldots, \phi_{k}\right)$ and $\theta_{2}=$ $\mathcal{X}\left(\psi_{1}, \ldots, \psi_{k}\right)$. Then $\theta_{1}$ and $\theta_{2}$ are $N_{\mathfrak{S}_{p^{k}}}\left(P_{p^{k}}\right)$-conjugate if and only if $\left\{i: \phi_{i}=\mathbb{1}_{P_{p}}\right\}=\{j$ : $\left.\psi_{j}=\mathbb{1}_{P_{p}}\right\}$.

Proof. This follows from a standard argument. We refer the reader to either [L19, Lemma 4.3] or to [G21, Corollary 2.5] for a complete proof.

In other words, $\mathcal{X}\left(\phi_{1}, \ldots, \phi_{k}\right)$ and $\mathcal{X}\left(\psi_{1}, \ldots, \psi_{k}\right)$ are $N_{\mathfrak{S}_{p^{k}}}\left(P_{p^{k}}\right)$-conjugate if and only if $\mathbb{1}_{P_{p}}$ occurs in exactly the same positions in the two sequences $\phi_{1}, \ldots, \phi_{k}$ and $\psi_{1}, \ldots, \psi_{k}$.

Next, we consider arbitrary natural numbers $n$. Suppose $n=\sum_{i=1}^{t} a_{i} p^{n_{i}}$ in $p$-adic expansion, as above. Since $P_{n} \cong\left(P_{p^{n_{1}}}\right)^{\times a_{1}} \times \cdots \times\left(P_{p^{n_{t}}}\right)^{\times a_{t}}$, we have

$$
\operatorname{Lin}\left(P_{n}\right)=\left(\operatorname{Lin}\left(P_{p^{n_{1}}}\right)\right)^{a_{1}} \times \cdots \times\left(\operatorname{Lin}\left(P_{p^{n_{t}}}\right)\right)^{a_{t}} .
$$

The case of $n=a p^{k}$ for $k \in \mathbb{N}$ and $a \in[p-1]$ is the most important for our purposes, since our goal is to prove Theorem 2.2. Therefore, while a statement analogous to Lemma 3.3 holds for all $n$, we need only record it here in the case $n=a p^{k}$. We note that $\operatorname{Lin}\left(P_{a p^{k}}\right)$ may be parametrised as follows:

$$
\operatorname{Lin}\left(P_{a p^{k}}\right)=\left\{\mathcal{L}\left(\phi_{1,1}, \phi_{1,2}, \ldots, \phi_{a, k-1}, \phi_{a, k}\right): \phi_{i, j} \in \operatorname{Lin}\left(P_{p}\right) \forall i \in[a], j \in[k]\right\}
$$

where $\mathcal{L}\left(\phi_{1,1}, \ldots, \phi_{a, k}\right)=\mathcal{X}\left(\phi_{1,1}, \ldots, \phi_{1, k}\right) \times \cdots \times \mathcal{X}\left(\phi_{a, 1}, \ldots, \phi_{a, k}\right)$.

Lemma 3.4. Let $k \in \mathbb{N}$ and let $a \in[p-1]$. Let $\theta_{1}, \theta_{2} \in \operatorname{Lin}\left(P_{a p^{k}}\right)$. Suppose $\theta_{1}=\mathcal{L}\left(\phi_{1,1}, \ldots, \phi_{a, k}\right)$ and $\theta_{2}=\mathcal{L}\left(\psi_{1,1}, \ldots, \psi_{a, k}\right)$. Then $\theta_{1}$ and $\theta_{2}$ are $N_{\mathfrak{S}_{n}}\left(P_{n}\right)$-conjugate if and only if there exists $\sigma \in \mathfrak{S}_{a}$ such that for each $i \in[a], \mathcal{X}\left(\phi_{i, 1}, \ldots, \phi_{i, k}\right)$ and $\mathcal{X}\left(\psi_{\sigma(i), 1}, \ldots, \psi_{\sigma(i), k}\right)$ are $N_{\mathfrak{S}_{p^{k}}}\left(P_{p^{k}}\right)-$ conjugate.

\footnotetext{
${ }^{1}$ We remark that $P_{p^{k}} / P_{p^{k}}{ }^{\prime}$ is isomorphic to $\left(P_{p}\right)^{k}$. Fixing a natural isomorphism $P_{p^{k}} / P_{p^{k}}{ }^{\prime} \cong\left(P_{p}\right)^{k}$, our indexing of $\operatorname{Lin}\left(P_{p^{k}}\right)$ may be obtained equivalently from the canonical bijection $\operatorname{Lin}\left(P_{p^{k}}\right) \rightarrow \operatorname{Irr}\left(P_{p^{k}} / P_{p^{k}}{ }^{\prime}\right)$.
} 
Proof. As with Lemma 3.3, we refer the reader to either [L19, Lemma 4.5] or to [G21, Lemma $2.14]$ for a complete proof.

In light of Lemmas 3.3 and 3.4 and our goal of proving Theorem 2.2. we may simplify the notation used to parametrise the linear characters of $P_{a p^{k}}$.

Notation 3.5. Let $k \in \mathbb{N}$ and $a \in[p-1]$. For the remainder of this article, we denote the elements of $\operatorname{Lin}\left(P_{a p^{k}}\right)$ as follows.

- Let $\mathbf{u}=\left(\mathbf{u}_{1}, \ldots, \mathbf{u}_{k}\right) \in\{0,1\}^{k}$. Then $\phi(\mathbf{u})$ denotes any element $\mathcal{X}\left(\psi_{1}, \ldots, \psi_{k}\right)$ of $\operatorname{Lin}\left(P_{p^{k}}\right)$ such that $\mathbf{u}_{j}=1$ if and only if $\psi_{j}=\mathbb{1}_{P_{p}}$.

- Let $\underline{\mathbf{u}}=\left(\mathbf{u}^{1}, \ldots, \mathbf{u}^{a}\right)$ with $\mathbf{u}^{i}=\left(\mathbf{u}_{1}^{i}, \ldots, \mathbf{u}_{k}^{i}\right) \in\{0,1\}^{k}$ for each $i \in[a]$. Then $\phi(\underline{\mathbf{u}})$ denotes any element $\mathcal{L}\left(\psi_{1,1}, \ldots, \psi_{a, k}\right)$ of $\operatorname{Lin}\left(P_{a p^{k}}\right)$ such that $\mathbf{u}_{j}^{i}=1$ if and only if $\psi_{i, j}=\mathbb{1}_{P_{p}}$.

For short, we will write $\phi(\mathbf{u}) \in \operatorname{Lin}\left(P_{p^{k}}\right)$ or $\phi(\underline{\mathbf{u}}) \in \operatorname{Lin}\left(P_{a p^{k}}\right)$, meaning that $\mathbf{u} \in\{0,1\}^{k}$ or $\underline{\mathbf{u}}=\left(\mathbf{u}^{1}, \ldots, \mathbf{u}^{a}\right)$ with $\mathbf{u}^{i} \in\{0,1\}^{k}$ for each $i \in[a]$ respectively.

With this, Theorem 2.2 is therefore equivalent to the following:

Theorem 3.6. Let $k \in \mathbb{N}$ and $a \in[p-1]$. Let $\phi(\underline{\mathbf{s}}), \phi(\underline{\mathbf{t}}) \in \operatorname{Lin}\left(P_{a p^{k}}\right)$. Suppose $\phi(\underline{\mathbf{s}}) \uparrow^{\mathfrak{S}_{a p^{k}}}=$ $\phi(\underline{\mathbf{t}}) \uparrow^{\mathfrak{S}_{a p^{k}}}$. Then there exists a permutation $\sigma \in \mathfrak{S}_{a}$ such that $\mathbf{s}^{i}=\mathrm{t}^{\sigma(i)}$ for all $i$.

\section{INDUCED CHARACTER VALUES}

Throughout this section, fix $p$ any prime and $k \in \mathbb{N}$. To complete the proof of Theorem $\mathrm{A}$ it remains to prove Theorem 3.6. Our approach will be to calculate the values $\phi(\underline{\mathbf{s}}) \uparrow^{\mathfrak{S}_{a p^{k}}(g)}$ for $g$ belonging to various different conjugacy classes of $\mathfrak{S}_{n}$, or in other words, for $g$ of various different cycle types. Our goal will be to express these values in terms of the sequences $\mathbf{s}^{i}$ of $\underline{\mathbf{s}}$, so that we can use the assumption $\phi(\underline{\mathbf{s}}) \uparrow^{\mathfrak{S}_{a p^{k}}}=\phi(\underline{\mathbf{t}}) \uparrow^{\mathfrak{S}_{a p^{k}}}$ to deduce information relating $\underline{\mathbf{s}}$ and t. Recall that

$$
\phi \uparrow_{P_{a p^{k}}}^{\mathfrak{S}_{a p^{k}}}(g)=\frac{\left|\mathbf{C}_{\mathfrak{S}_{a p^{k}}}(g)\right|}{\left|P_{a p^{k}}\right|} \cdot \sum_{x \in \operatorname{ccl}_{\mathfrak{S}_{a p^{k}}}(g) \cap P_{a p^{k}}} \phi(x) .
$$

Here and throughout, $\mathbf{C}_{G}(g)$ denotes the centraliser and $\operatorname{ccl}_{G}(g)$ the conjugacy class in $G$ of an element $g \in G$. Since it is easy to calculate $\left|\mathbf{C}_{\mathfrak{S}_{a p^{k}}}(g)\right|$ and $\left|P_{a p^{k}}\right|$, our focus is on understanding the summation term in (11), and therefore the structure of $\operatorname{ccl}_{\mathfrak{S}_{a p^{k}}}(g) \cap P_{a p^{k}}$. By writing $P_{a p^{k}}$ as the direct product of $a$ copies of $P_{p^{k}}$, we can reduce this task to understanding $\operatorname{ccl}_{\mathfrak{S}_{p^{k}}}(h) \cap P_{p^{k}}$ for various $h \in \mathfrak{S}_{p^{k}}$. Since $P_{p^{k}}=P_{p^{k-1}} \prec P_{p}$, we can tackle this by induction on $k$. However, the wreath product structure makes this a rather complicated task in general.

Fortunately, in order to deduce Theorem 3.6, it will in fact suffice to consider $\phi(\underline{\mathbf{s}})^{\uparrow^{\mathfrak{S}}{ }_{a p^{k}}(g)}$ for only those $g$ which, excluding fixed points, are products of cycles whose lengths are distinct powers of $p$. In this case the calculations simplify considerably, as explained in the following remark.

Remark 4.1. The reason for the aforementioned simplification is exemplified by the case $n=p^{k}$. Given an element $g \in \mathfrak{S}_{p^{k}}$ which, excluding fixed points, is a product of disjoint cycles of distinct $p$-power length, either $g$ has at least one fixed point in $\left[p^{k}\right]$ or $g$ is a single $p^{k}$-cycle. In the first case, the presence of a fixed point implies that $\operatorname{ccl}_{\mathfrak{S}_{p^{k}}}(g) \cap P_{p^{k}}$ is contained in the natural subgroup $P_{p^{k-1}} \times \cdots \times P_{p^{k-1}}$ of $P_{p^{k}}$, allowing us to avoid working with the wreath product structure. The second case will be dealt with using Lemma 3.2, which characterises $p^{k}$-cycles in $P_{p^{k}}$. 
The structure of this section is as follows. We begin with the case $n=p^{k}$ and $g$ a single $p^{l}$-cycle, for some $1 \leq l \leq k$. In this case, the value $\phi \uparrow_{P_{p^{k}}}^{\mathfrak{S}_{p^{k}}}(g)$ (for $\phi \in \operatorname{Lin}\left(P_{p^{k}}\right)$ ) is given by Lemma 4.4. This lemma will be proved by induction on $k$, with the key ideas as described in the paragraph above. In Proposition 4.9, we extend these methods to any $g \in \mathfrak{S}_{p^{k}}$ a product of disjoint cycles of distinct $p$-power length. Theorem 4.13 then generalises Proposition 4.9 from $n=p^{k}$ to $n=a p^{k}$ with $a \in[p-1]$ by using the fact that $P_{a p^{k}}=P_{p^{k}} \times \cdots \times P_{p^{k}}$. This is conceptually straightforward, but there are several combinatorial simplifications needed to reduce to a usable formula.

We are now ready to begin the technical details. We start with some definitions which will help ease the notation in the rest of this section.

Definition 4.2. Let $b \in \mathbb{N}$ and suppose that $l_{1}, l_{2}, \ldots, l_{b}$ are natural numbers in $[k]$. Suppose $g \in \mathfrak{S}_{p^{k}}$ has disjoint cycles of length $p^{l_{1}}, p^{l_{2}}, \cdots, p^{l_{b}}, 1, \ldots, 1$ (we also say $g$ has cycle type $\left.p^{l_{1}} p^{l_{2}} \cdots p^{l_{b}}\right)$. Let $\mathbf{u} \in\{0,1\}^{k}$. Define

$$
\Gamma_{l_{1}, l_{2}, \ldots, l_{b} ; k}(\mathrm{u}):=\sum_{x \in \operatorname{ccl}_{\mathfrak{S}_{p^{k}}}(g) \cap P_{p^{k}}} \phi(\mathrm{u})(x) .
$$

More generally, we define $\Gamma_{l_{1}, l_{2}, \ldots, l_{b} ; k}(\mathrm{u})$ for any natural numbers $l_{1}, \ldots, l_{b}$ by setting the value to be 0 whenever $p^{l_{1}}+p^{l_{2}}+\cdots+p^{l_{b}}>p^{k}$. In particular, (1) can be rewritten as

$$
\phi(\mathbf{u}) \uparrow_{P_{p^{k}}}^{\mathfrak{S}_{p^{k}}}(g)=\frac{\left|\mathbf{C}_{\mathfrak{G}_{p^{k}}}(g)\right|}{\left|P_{p^{k}}\right|} \cdot \Gamma_{l_{1}, \ldots, l_{b} ; k}(\mathbf{u}) .
$$

Definition 4.3. (i) Let $l \in[k]$ and $\mathbf{u}=\left(\mathrm{u}_{1}, \ldots, \mathrm{u}_{k}\right) \in\{0,1\}^{k}$. We define

$$
C_{l}(\mathrm{u}):=p^{\frac{p^{l}-1}{p-1}-2 l} \cdot \prod_{m=1}^{l}\left(p \mathbf{u}_{m}-1\right) .
$$

(ii) For a multiset $T$ of natural numbers, we let $X(T)$ (respectively $Y(T)$ ) denote the set of elements of $P_{p^{k}}$ (respectively $P_{p^{k-1}}$ ) of cycle type $\prod_{t \in T} p^{t}$. If $T=\left\{t_{1}, \ldots, t_{z}\right\}$, we sometimes also use the notation $X\left(t_{1}, \ldots, t_{z}\right)$ or $Y\left(t_{1}, \ldots, t_{z}\right)$.

It is useful to observe that $C_{l}(\mathrm{u})$ does not depend on $\mathrm{u}_{l+1}, \ldots, \mathrm{u}_{k}$. In other words, $C_{l}(\mathrm{u})=$ $C_{l}\left(\left(\mathbf{u}_{1}, \ldots, \mathbf{u}_{l}\right)\right)$. The following lemma gives the value of $\phi(\mathrm{u}) \uparrow_{P_{p^{k}}}^{\mathfrak{S}_{p^{k}}}$ on a single $p^{l}$-cycle, using (2).

Lemma 4.4. Let $l \in[k]$ and let $\mathrm{u}=\left(\mathrm{u}_{1}, \ldots, \mathrm{u}_{k}\right) \in\{0,1\}^{k}$. Then

$$
\Gamma_{l ; k}(\mathbf{u})=p^{k} \cdot C_{l}(\mathbf{u}) \text {. }
$$

Proof. Observe that if $l=k=1$, then $\Gamma_{1 ; 1}(\mathbf{u})=p \mathbf{u}_{1}-1$. In other words,

$$
\Gamma_{1 ; 1}(\mathbf{u})=\sum_{x \in P_{p} \backslash\{1\}} \phi(\mathbf{u})(x)= \begin{cases}p-1 & \text { if } \phi(\mathbf{u})=\mathbb{1}_{P_{p}} \quad(\text { i.e. if } \mathbf{u}=(1)), \\ -1 & \text { otherwise } \quad \text { (i.e. if } \mathbf{u}=(0)) .\end{cases}
$$

Now let $k \geq 2$ and let $\mathbf{u}^{-}=\left(\mathbf{u}_{1}, \ldots, \mathbf{u}_{k-1}\right)$. First suppose $l=k$. Let $x \in X(k)$, that is, let $x$ be a $p^{k}$-cycle in $P_{p^{k}}$. By Lemma 3.2, $x=\left(f_{1}, \ldots, f_{p} ; \sigma\right)$ where $\sigma \in P_{p} \backslash\{1\}, f_{i} \in P_{p^{k-1}}$ for all $i$ and $f_{\sigma^{p-1}(1)} \cdots f_{\sigma(1)} \cdot f_{1} \in Y(k-1)$. By Lemma 3.1.

$$
\Gamma_{k ; k}(\mathbf{u})=\sum_{x \in X(k)} \mathcal{X}\left(\mathbf{u}^{-} ; \mathbf{u}_{k}\right)\left(\left(f_{1}, \ldots, f_{p} ; \sigma\right)\right)=\sum_{x \in X(k)} \phi\left(\mathbf{u}^{-}\right)\left(f_{\sigma^{p-1}(1)} \cdots f_{\sigma(1)} \cdot f_{1}\right) \cdot \phi\left(\mathbf{u}_{k}\right)(\sigma)
$$




$$
=\left|P_{p^{k-1}}\right|^{p-1} \cdot \sum_{y \in Y(k-1)} \phi\left(\mathbf{u}^{-}\right)(y) \cdot \sum_{\sigma \in P_{p} \backslash\{1\}} \phi\left(\mathbf{u}_{k}\right)(\sigma)=p^{p^{k-1}-1} \cdot \Gamma_{k-1 ; k-1}\left(\mathbf{u}^{-}\right) \cdot\left(p \mathbf{u}_{k}-1\right) .
$$

The third equality holds since for any fixed $y \in Y(k-1)$, we may choose the $p-1$ elements $f_{1}, f_{\sigma(1)}, \ldots, f_{\sigma^{p-2}(1)}$ in $P_{p^{k-1}}$ freely, after which $f_{\sigma^{p-1}(1)} \cdots f_{\sigma(1)} \cdot f_{1}=y$ uniquely determines $f_{\sigma^{p-1}(1)}$. Inductively, we have that

$$
\Gamma_{k ; k}(\mathrm{u})=p^{\left(p^{k-1}+p^{k-2}+\cdots+1\right)-k} \cdot \prod_{m=1}^{k}\left(p \mathbf{u}_{m}-1\right)=p^{\frac{p^{k}-1}{p-1}-k} \cdot \prod_{m=1}^{k}\left(p \mathbf{u}_{m}-1\right) .
$$

Next, let $l \in[k-1]$. Let $x \in X(l)$. Then $x$ must have a fixed point as $l<k$. Thus $x=$ $\left(f_{1}, \ldots, f_{p} ; 1\right)$ where $f_{i} \in Y(l)$ for a unique $i \in[p]$ and $f_{j}=1$ for all $j \neq i$. Hence by Lemma 3.1,

$$
\begin{aligned}
\Gamma_{l ; k}(\mathbf{u}) & =\sum_{x \in X(l)} \phi\left(\mathbf{u}^{-}\right)\left(f_{1}\right) \cdots \phi\left(\mathbf{u}^{-}\right)\left(f_{p}\right) \cdot \phi\left(\mathbf{u}_{k}\right)(1) \\
& =\left(\begin{array}{c}
p \\
1
\end{array}\right) \sum_{y \in Y(l)} \phi\left(\mathbf{u}^{-}\right)(y)=p \cdot \Gamma_{l ; k-1}\left(\mathbf{u}^{-}\right)=p^{k-l} \cdot \Gamma_{l ; l}\left(\left(\mathbf{u}_{1}, \ldots, \mathbf{u}_{l}\right)\right) .
\end{aligned}
$$

The assertion then follows from the $l=k$ case.

Corollary 4.5. Let $a \in[p-1]$ and $\phi \in \operatorname{Lin}\left(P_{a p^{k}}\right)$. Then $\phi \uparrow_{P_{a p^{k}}}^{\mathfrak{S}_{a p^{k}}}(g) \neq 0$ where $g$ is a product of a disjoint $p^{k}$-cycles.

Proof. From (1), it suffices to show that

$$
\sum_{x \in \operatorname{ccl}_{\mathfrak{S}_{a p^{k}}}(g) \cap P_{a p^{k}}} \phi(x) \neq 0 .
$$

Since $P_{a p^{k}}=P_{p^{k}} \times \cdots \times P_{p^{k}}$ ( $a$ times), $x \in P_{a p^{k}}$ implies $x=x_{1} \cdots x_{a}$ with each $x_{i}$ in a distinct direct factor $P_{p^{k}}$. Letting $\phi=\phi\left(\mathbf{u}^{1}\right) \times \cdots \times \phi\left(\mathbf{u}^{a}\right)$ where $\phi\left(\mathbf{u}^{i}\right) \in \operatorname{Lin}\left(P_{p^{k}}\right)$ for each $i$, we therefore have that

$$
\sum_{x \in \operatorname{ccl}_{\mathfrak{S}_{a p^{k}}}(g) \cap P_{a p^{k}}} \phi(x)=\prod_{i=1}^{a}\left(\sum_{x_{i} \in X(k)} \phi\left(\mathbf{u}^{i}\right)\left(x_{i}\right)\right)=\prod_{i=1}^{a} \Gamma_{k ; k}\left(\mathbf{u}^{i}\right)=\prod_{i=1}^{a}\left(p^{k} \cdot C_{k}\left(\mathbf{u}^{i}\right)\right)
$$

by Lemma 4.4, which is non-zero (Definition 4.3).

Remark 4.6. Lemma 4.4 is already enough to prove Theorem 3.6 when $a=1$. Indeed, let $\phi(\mathrm{s})$, $\phi(\mathrm{t}) \in \operatorname{Lin}\left(P_{p^{k}}\right)$ for some $\mathbf{s}, \mathrm{t} \in\{0,1\}^{k}$ and suppose $\phi(\mathbf{s}) \uparrow^{\mathfrak{S}_{p^{k}}}=\phi(\mathrm{t}) \uparrow^{\mathfrak{S}_{p^{k}}}$. Then $\phi(\mathbf{s}) \uparrow^{\mathfrak{S}_{p^{k}}}(g)=$ $\phi(\mathrm{t}) \uparrow^{\mathfrak{S}_{p^{k}}}(g)$ for each $g \in \mathfrak{S}_{p^{k}}$, in particular $g$ of cycle type $p^{l}$ for each $l \in[k]$. By Lemma 4.4, this implies

$$
\prod_{m=1}^{l}\left(p \mathbf{s}_{m}-1\right)=\prod_{m=1}^{l}\left(p \mathbf{t}_{m}-1\right)
$$

for all $l \in[k]$. Therefore $\mathbf{s}_{m}=\mathrm{t}_{m}$ for all $m \in[k]$, and thus $\mathrm{s}=\mathrm{t}$.

However, Lemma4.4 is not enough when $a>1$. For example, let $a=2, k=3$ and consider $\underline{\mathbf{s}}=$ $((1,0,0),(0,1,1))$ and $\underline{\mathbf{t}}=((1,0,1),(0,1,0))$. The induced characters $\phi(\underline{\mathbf{s}})^{\uparrow} \widetilde{\mathfrak{S}}_{2 p^{3}}$ and $\phi(\underline{\mathbf{t}}) \uparrow^{\widetilde{\mathfrak{S}}_{2 p^{3}}}$ agree on $p, p^{2}$ and $p^{3}$-cycles, but are not equal. This motivates considering more complicated cycle types, as we shall now do. 
The next proposition gives the value of the induced character $\phi(\mathbf{u}) \uparrow_{P_{p^{k}}}^{\mathfrak{S}_{p^{k}}}$ on a product of $p$-power cycles of distinct length. Before we state the proposition, we give some key definitions.

Notation 4.7. (i) For a set $A$, let Part $A$ denote the set of partitions of $A$, e.g.

Part $\{1,2,3\}=\{\{\{1,2,3\}\},\{\{1,2\},\{3\}\},\{\{1,3\},\{2\}\},\{\{2,3\},\{1\}\},\{\{1\},\{2\},\{3\}\}\}$.

(ii) For the remainder of Section 4 , suppose $l_{1}, l_{2}, \ldots, l_{b}$ are fixed integers for some $b \in \mathbb{N}$ such that $1 \leq l_{1}<l_{2}<\cdots<l_{b} \leq k$. We further let $p_{j}:=p^{l_{j}}$.

Definition 4.8. Let $\tau=\left\{t_{1}<t_{2}<\cdots<t_{z}\right\} \subseteq[b]$ be a set of distinct natural numbers. Let $i \in \mathbb{N}$. Define

$$
R_{i}^{\tau}:= \begin{cases}\left(p^{i}-p_{t_{z}}\right)\left(p^{i}-p_{t_{z}}-p_{t_{z-1}}\right) \cdots\left(p^{i}-p_{t_{z}}-\cdots-p_{t_{2}}\right) & \text { if } z \geq 2 \\ 1 & \text { if } z=1\end{cases}
$$

For example, if $b>1$ then $R_{k}^{[b]}=\left(p^{k}-p^{l_{b}}\right)\left(p^{k}-p^{l_{b}}-p^{l_{b-1}}\right) \cdots\left(p^{k}-p^{l_{b}}-p^{l_{b-1}}-\cdots-p^{l_{2}}\right)$.

Proposition 4.9. Let $b \in[p-1]$ and $\mathrm{u} \in\{0,1\}^{k}$. Suppose $l_{1}, l_{2}, \ldots, l_{b}$ are integers such that $1 \leq l_{1}<l_{2}<\cdots<l_{b} \leq k$. Then

$$
\Gamma_{l_{1}, l_{2}, \ldots, l_{b} ; k}(\mathrm{u})=p^{k} \cdot C_{l_{1}}(\mathrm{u}) \cdots C_{l_{b}}(\mathrm{u}) \cdot R_{k}^{[b]} .
$$

In Appendix $\mathrm{A}$, we provide detailed examples illustrating the proof of Proposition 4.9 for $b=2$ and $b=3$. In Example A.1 we prove the proposition directly for $b=2$, and in Example A.2 we demonstrate the inductive step of the argument by deducing the proposition for $b=3$ from the case of $b=2$. Equations (4.9)(a)-(g)) below are labelled as such to illustrate their correspondence to equations A.2 (a) $-(\mathrm{g}))$ in Appendix A. It may help the reader to first familiarise with the main proof ideas by reading Examples A.1 and A.2 before reading the general proof, which we now give.

Proof of Proposition 4.9. We proceed by induction on $b$, with Lemma 4.4 providing the base case $b=1$. Now assume $b \geq 2$, and that Proposition 4.9 holds for all $b^{\prime} \in[p-1]$ such that $b^{\prime}<b$. Notice that both sides of (3) equal 0 if $l_{b}=k$, so from now on we may assume $l_{b}<k$. Let $\mathbf{u}^{-}=\left(\mathbf{u}_{1}, \ldots, \mathbf{u}_{k-1}\right)$. From Definitions 4.2 and 4.3 ,

$$
\Gamma_{l_{1}, \ldots, l_{b} ; k}(\mathrm{u})=\sum_{x \in X\left(l_{1}, \ldots, l_{b}\right)} \phi(\mathrm{u})(x) .
$$

Consider some $x \in X\left(l_{1}, \ldots, l_{b}\right)$. Since $l_{1}<l_{2}<\cdots<l_{b}<k$, we deduce that $x$ has a fixed point. Hence $x=\left(f_{1}, \ldots, f_{p} ; 1\right)$ with $f_{1}, \ldots, f_{p} \in P_{p^{k-1}}$ and so

$$
\phi(\mathbf{u})(x)=\phi\left(\mathbf{u}^{-}\right)\left(f_{1}\right) \cdot \phi\left(\mathbf{u}^{-}\right)\left(f_{2}\right) \cdots \phi\left(\mathbf{u}^{-}\right)\left(f_{p}\right)
$$

by Lemma 3.1. Moreover, the product of the cycle types of $f_{i}$ over all $i$ equals $p^{l_{1}} \cdots p^{l_{b}}$ since $x \in X\left(l_{1}, \ldots, l_{b}\right)$. By considering how the cycle lengths $p^{l_{w}}(w \in[b])$ are grouped together to give the cycle types of $f_{1}, \ldots, f_{p}$, we can rewrite the sum over $x \in X\left(l_{1}, \ldots, l_{b}\right)$ in (4.9)(a) as a sum over partitions $\nu$ of the set $[b]=\{1,2, \ldots, b\}$ to obtain

$$
\begin{aligned}
\Gamma_{l_{1}, \ldots, l_{b} ; k}(\mathbf{u}) & =\sum_{\nu \in \operatorname{Part}[b]} \frac{p !}{(p-|\nu|) !} \cdot \prod_{\omega \in \nu} \Gamma_{l_{\omega} ; k-1}\left(\mathbf{u}^{-}\right) \\
& =\sum_{\substack{\nu \in \operatorname{Part}[b] \\
\nu \neq\{[b]\}}} \frac{p !}{(p-|\nu|) !} \cdot \prod_{\omega \in \nu} \Gamma_{l_{\omega} ; k-1}\left(\mathbf{u}^{-}\right)+p \cdot \Gamma_{l_{1}, \ldots, l_{b} ; k-1}\left(\mathbf{u}^{-}\right) .
\end{aligned}
$$


Here $\Gamma_{l_{\omega} ; k-1}\left(\mathbf{u}^{-}\right)$denotes $\Gamma_{l_{w_{1}}, \ldots, l_{w_{t}} ; k-1}\left(\mathbf{u}^{-}\right)$when $\omega=\left\{w_{1}, \ldots, w_{t}\right\} \subseteq[b]$. (So the last term of (4.9(b)] which corresponds to $\nu=\{[b]\}$ can also be written as $p \cdot \Gamma_{l_{[b]} ; k-1}\left(\mathbf{u}^{-}\right)$, for instance.) By iterating (4.9(b) we obtain the following:

$$
\Gamma_{l_{1}, \ldots, l_{b} ; k}(\mathbf{u})=\sum_{i=l_{b}}^{k-1} p^{k-i} \sum_{\substack{\nu \in \operatorname{Part}[b] \\ \nu \neq\{[b]\}}} \frac{(p-1) !}{(p-|\nu|) !} \cdot \prod_{\omega \in \nu} \Gamma_{l_{\omega} ; i}\left(\left(\mathbf{u}_{1}, \ldots, \mathbf{u}_{i}\right)\right),
$$

recalling that $\Gamma_{l_{1}, l_{2}, \ldots, l_{b} ; i}=0$ if $l_{b}=i$. Since $\nu \neq\{[b]\}$, every $\omega$ appearing in (4.9)(c) satisfies $|\omega|<b$. Therefore the inductive hypothesis gives

$$
\Gamma_{l_{\omega} ; i}\left(\left(\mathbf{u}_{1}, \ldots, \mathbf{u}_{i}\right)\right)=p^{i} \cdot C_{l_{w_{1}}}(\mathbf{u}) \cdots C_{l_{w_{t}}}(\mathrm{u}) \cdot R_{i}^{\omega} .
$$

Substituting this into (4.9(c) $)$, we obtain

$$
\begin{aligned}
\Gamma_{l_{1}, \ldots, l_{b} ; k}(\mathbf{u}) & =\sum_{i=l_{b}}^{k-1} p^{k-i} \sum_{\substack{\nu \in \operatorname{Part}[b] \\
\nu \neq\{[b]\}}} \frac{(p-1) !}{(p-|\nu|) !} \cdot \prod_{\omega \in \nu}\left[p^{i} \cdot \prod_{w \in \omega} C_{l_{w}}(\mathbf{u}) \cdot R_{i}^{\omega}\right] \\
& =p^{k} \cdot \prod_{i=1}^{b} C_{l_{i}}(\mathbf{u}) \cdot \sum_{i=l_{b}}^{k-1} \sum_{\substack{\nu \in \operatorname{Part}[b] \\
\nu \neq\{[b]\}}} \frac{(p-1) !}{(p-|\nu|) !} \cdot p^{i(|\nu|-1)} \cdot \prod_{\omega \in \nu} R_{i}^{\omega} .
\end{aligned}
$$

Thus, it remains to prove that $\Sigma_{b}=R_{k}^{[b]}$, where we define $\Sigma_{b}$ as follows:

$$
\Sigma_{b}:=\sum_{i=l_{b}}^{k-1} \sum_{\substack{\nu \in \text { Part }[b] \\ \nu \neq\{[b]\}}} \frac{(p-1) !}{(p-|\nu|) !} \cdot p^{i(|\nu|-1)} \cdot \prod_{\omega \in \nu} R_{i}^{\omega}
$$

Let

$$
Q_{i}:=\sum_{\substack{\gamma \in \operatorname{Part}\{2,3, \ldots, b\} \\ \gamma \neq\{\{2,3, \ldots, b\}\}}} \frac{(p-1) !}{(p-|\gamma|) !} \cdot p^{i(|\gamma|-1)} \cdot \prod_{\omega \in \gamma} R_{i}^{\omega} .
$$

Since Proposition 4.9 holds for $b-1$ (for $l_{2}<l_{3}<\cdots<l_{b}$ ), we have for all $i \in \mathbb{N}$ such that $i>l_{b}$ the following:

$$
\sum_{j=l_{b}}^{i-1} Q_{j}=\left(p^{i}-p^{l_{b}}\right)\left(p^{i}-p^{l_{b}}-p^{l_{b-1}}\right) \cdots\left(p^{i}-p^{l_{b}}-\cdots-p^{l_{3}}\right)=R_{i}^{\{2,3, \ldots, b\}} .
$$

Now we return to $\Sigma_{b}$, and sum together the terms whose associated $\nu \in$ Part $[b]$ correspond to the same $\gamma \in$ Part $\{2,3, \ldots, b\}$ upon removing the number 1 . Moreover, for a fixed $\gamma$, we first group together those terms with $\nu$ of the form (a) $\nu=(\gamma \backslash\{\tau\}) \cup\{\tau \cup\{1\}\}$ for some $\tau \in \gamma$, and separately (b) $\nu=\gamma \cup\{\{1\}\}$. Notice $|\nu|=|\gamma|$ or $|\nu|=|\gamma|+1$ respectively. In particular, in case (a), letting $\tau=\left\{w_{1}, \cdots, w_{t}\right\} \subseteq\{2,3, \ldots, b\}$ then

$$
\prod_{\omega \in \nu} R_{i}^{\omega}=\prod_{\omega \in \gamma} R_{i}^{\omega} \cdot\left(p^{i}-p^{l_{w_{t}}}-p^{l_{w_{t-1}}}-\cdots-p^{l_{w_{1}}}\right),
$$

while in case (b) we have $\prod_{\omega \in \nu} R_{i}^{\omega}=\prod_{\omega \in \gamma} R_{i}^{\omega}$. Finally, observe that since $\nu \in$ Part [b] with $\nu \neq\{[b]\}$, then the only $\nu$ whose corresponding $\gamma$ equals $\{2,3, \ldots, b\}$ is $\nu=\{\{1\},\{2,3, \ldots, b\}\}$. 
Letting $B=\{2,3, \ldots, b\}$, we thus obtain

$$
\begin{aligned}
& \Sigma_{b}=\sum_{i=l_{b}}^{k-1}\left[\sum_{\substack{\gamma \in \operatorname{Part} B \\
\gamma \neq\{B\}}}(\underbrace{\frac{(p-1) !}{(p-|\gamma|) !} \cdot p^{i(|\gamma|-1)} \cdot \prod_{\omega \in \gamma} R_{i}^{\omega} \cdot \sum_{\tau \in \gamma}\left(p^{i}-\sum_{w \in \tau} p^{l_{w}}\right)}_{\nu=(\gamma \backslash\{\tau\}) \cup\{\tau \cup\{1\}\} \text { for some } \tau \in \gamma}+\underbrace{\frac{(p-1) !}{(p-|\gamma|-1) !} \cdot p^{i|\gamma|} \cdot \prod_{\omega \in \gamma} R_{i}^{\omega}}_{\nu=\gamma \cup\{\{1\}\}})\right. \\
& +\underbrace{\frac{(p-1) !}{(p-2) !} \cdot p^{i} \cdot R_{i}^{\{2,3, \ldots, b\}}}_{\gamma=\{B\}}] \\
& =\sum_{i=l_{b}}^{k-1}\left[\sum_{\substack{\gamma \in \operatorname{Part} B \\
\gamma \neq\{B\}}} \frac{(p-1) ! \cdot p^{i(|\gamma|-1)}}{(p-|\gamma|) !} \prod_{\omega \in \gamma} R_{i}^{\omega}\left(\sum_{\tau \in \gamma}\left(p^{i}-\sum_{w \in \tau} p^{l_{w}}\right)+(p-|\gamma|) p^{i}\right)+(p-1) p^{i} R_{i}^{\{2, \ldots, b\}}\right] \\
& =\sum_{i=l_{b}}^{k-1}\left[Q_{i} \cdot\left(p^{i+1}-p^{l_{b}}-\cdots-p^{l_{2}}\right)+(p-1) p^{i} \cdot R_{i}^{\{2,3, \ldots, b\}}\right] \\
& \left.=\left(-p^{l_{b}}-\cdots-p^{l_{2}}\right) \sum_{i=l_{b}}^{k-1} Q_{i}+\sum_{i=l_{b}}^{k-1}\left[p^{i+1} Q_{i}+(p-1) p^{i} \sum_{j=l_{b}}^{i-1} Q_{j}\right] \quad(\text { by } \overline{4.9(\mathrm{f})})\right) \\
& =\left(-p^{l_{b}}-\cdots-p^{l_{2}}\right) \sum_{i=l_{b}}^{k-1} Q_{i}+p^{k} \cdot \sum_{h=l_{b}}^{k-1} Q_{h} \\
& =\left(p^{k}-p^{l_{b}}-\cdots-p^{l_{2}}\right) \cdot\left(p^{k}-p^{l_{b}}\right)\left(p^{k}-p^{l_{b}}-p^{l_{b-1}}\right) \cdots\left(p^{k}-p^{l_{b}}-\cdots-p^{l_{3}}\right),
\end{aligned}
$$

which concludes the proof.

The final proposition of this section gives the value of the induced character $\phi(\underline{\mathbf{u}}) \uparrow_{P_{a p^{k}}}^{\mathfrak{S}_{a p^{k}}}$ on a product of $p$-power cycles of distinct length where $a \in[p-1]$. We first fix some useful notation.

Definition 4.10. Suppose $a \in[p-1]$ is fixed. For a multiset $T=\left\{t_{1}, \ldots, t_{z}\right\}$ of natural numbers, we let $W(T)$ (or $W\left(t_{1}, \ldots, t_{z}\right)$ ) denote the set of elements of $P_{a p^{k}}$ of cycle type $\prod_{t \in T} p^{t}$.

Notation 4.11. For the remainder of Section 4 , we fix the following notation. Recall $l_{1}, l_{2}, \ldots, l_{b}$ are fixed integers such that $1 \leq l_{1}<l_{2}<\cdots<l_{b} \leq k$. Suppose further that $b \in[a]$ and $a \in[p-1]$. Fix $\phi(\underline{\mathbf{u}}) \in \operatorname{Lin}\left(P_{a p^{k}}\right)$ with $\underline{\mathbf{u}}=\left(\mathbf{u}^{1}, \ldots, \mathbf{u}^{a}\right)$ and $\mathbf{u}^{i} \in\{0,1\}^{k}$ for each $i$.

For the function $C_{l}(\mathrm{u})$ introduced in Definition 4.3 (i), we simplify the notation by making the following abbreviations:

$$
C_{t}(i):=C_{l_{t}}\left(\mathrm{u}^{i}\right) \quad \text { and } \quad C_{r} C_{s} \cdots C_{t}(i):=C_{r}(i) \cdot C_{s}(i) \cdots C_{t}(i) .
$$

Furthermore, we let

$$
\left(\sum C_{r} C_{s} \cdots C_{t}\right):=\sum_{i=1}^{a} C_{r} C_{s} \cdots C_{t}(i)
$$

when the summation index runs through all of $\{1,2, \ldots, a\}$.

Definition 4.12. Recall that $p_{j}:=p^{l_{j}}$. For $\tau=\left\{t_{1}<t_{2}<\cdots<t_{z}\right\} \subseteq[b]$ a set of distinct natural numbers, we write

$$
M(\tau):= \begin{cases}p_{t_{z}} \cdot\left(p_{t_{z}}+p_{t_{z-1}}\right) \cdots\left(p_{t_{z}}+\cdots+p_{t_{2}}\right) & \text { if } z \geq 2 \\ 1 & \text { if } z=1\end{cases}
$$


Theorem 4.13. Using Notation 4.11, we let $g \in \mathfrak{S}_{a p^{k}}$ be of cycle type $\left(p^{l_{1}}, p^{l_{2}}, \ldots, p^{l_{b}}\right)$ and we set $\mathbf{C}=\mathbf{C}_{\mathfrak{S}_{a p k}}(g)$. Then we have that

$$
\frac{\left|P_{a p^{k}}\right|}{|\mathbf{C}|} \cdot \phi(\underline{\mathbf{u}}) \uparrow^{\mathfrak{S}_{a p^{k}}}(g)=\sum_{\nu \in \operatorname{Part}[b]}(-1)^{b-|\nu|} \cdot p^{k|\nu|} \cdot \prod_{\tau \in \nu}\left[M(\tau) \cdot \sum_{i=1}^{a}\left(\prod_{t \in \tau} C_{t}(i)\right)\right] .
$$

Before starting with the proof of Theorem 4.13, we remark that since

$$
\frac{\left|P_{a p^{k}}\right|}{|\mathbf{C}|} \cdot \phi(\underline{\mathbf{u}}) \uparrow^{\mathfrak{S}_{a p^{k}}}(g)=\sum_{x \in W\left(l_{1}, \ldots, l_{b}\right)} \phi(\underline{\mathbf{u}})(x),
$$

our main aim in the next few pages will be to show that

$$
\sum_{x \in W\left(l_{1}, \ldots, l_{b}\right)} \phi(\underline{\mathbf{u}})(x)=\sum_{\nu \in \operatorname{Part}[b]}(-1)^{b-|\nu|} \cdot p^{k|\nu|} \cdot \prod_{\tau \in \nu}\left[M(\tau) \cdot \sum_{i=1}^{a}\left(\prod_{t \in \tau} C_{t}(i)\right)\right] .
$$

For example, when $b=3$, the equation above (and more generally Theorem 4.13) asserts that

$$
\begin{array}{r}
\sum_{x \in W\left(l_{1}, l_{2}, l_{3}\right)} \phi(\underline{\mathbf{u}})(x)=p^{3 k}\left(\sum C_{1}\right)\left(\sum C_{2}\right)\left(\sum C_{3}\right)-p^{2 k} \cdot p_{2}\left(\sum C_{1} C_{2}\right)\left(\sum C_{3}\right)-p^{2 k} \cdot p_{3}\left(\sum C_{1} C_{3}\right)\left(\sum C_{2}\right) \\
-p^{2 k} \cdot p_{3}\left(\sum C_{2} C_{3}\right)\left(\sum C_{1}\right)+p^{k} \cdot p_{3}\left(p_{3}+p_{2}\right)\left(\sum C_{1} C_{2} C_{3}\right) .
\end{array}
$$

We remark that the case of $a=1$ follows immediately from Lemma 4.4, so from now on we may assume $a \geq 2$. We now begin the proof of Theorem 4.13, which forms the remainder of this section.

4.1. Proof of Theorem 4.13. Let $x \in W\left(l_{1}, \ldots, l_{b}\right)$. Since $P_{a p^{k}} \cong P_{p^{k}} \times \cdots \times P_{p^{k}}$ (a times), we can write $x=x_{1} \cdots x_{a}$ with each $x_{i}$ in a distinct direct factor $P_{p^{k}}$. Then $\phi(\underline{\mathbf{u}})(x)=$ $\phi\left(\mathbf{u}^{1}\right)\left(x_{1}\right) \cdots \phi\left(\mathbf{u}^{a}\right)\left(x_{a}\right)$. Since $x \in W\left(l_{1}, \ldots, l_{b}\right)$, the cycle types of $x_{1}, \ldots, x_{a}$ naturally give a partition of the set $[b]=\{1,2, \ldots, b\}$. We regroup the sum over $x \in W\left(l_{1}, \ldots, l_{b}\right)$ according to the corresponding partition of $[b]$ and substitute in Proposition 4.9 , in order to express $\sum_{x} \phi(\underline{\mathbf{u}})(x)$ in terms of the function $C_{t}(i)$.

For example, when $b=3$ we have (recall the five partitions of the set [3])

$$
\begin{aligned}
& \sum_{x \in W\left(l_{1}, l_{2}, l_{3}\right)} \phi(\underline{\mathbf{u}})(x)= \sum_{\substack{h, i, j \in[a] \\
\text { distinct }}} \Gamma_{l_{1} ; k}\left(\mathbf{u}^{h}\right) \Gamma_{l_{2} ; k}\left(\mathbf{u}^{i}\right) \Gamma_{l_{3} ; k}\left(\mathbf{u}^{j}\right)+\sum_{i \neq j \in[a]}\left[\Gamma_{l_{1}, l_{2} ; k}\left(\mathbf{u}^{i}\right) \Gamma_{l_{3} ; k}\left(\mathbf{u}^{j}\right)\right. \\
&\left.+\Gamma_{l_{1}, l_{3} ; k}\left(\mathbf{u}^{i}\right) \Gamma_{l_{2} ; k}\left(\mathbf{u}^{j}\right)+\Gamma_{l_{2}, l_{3} ; k}\left(\mathbf{u}^{i}\right) \Gamma_{l_{1} ; k}\left(\mathbf{u}^{j}\right)\right]+\sum_{i=1}^{a} \Gamma_{l_{1}, l_{2}, l_{3} ; k}\left(\mathbf{u}^{i}\right) \\
&=p^{3 k} \sum_{\substack{h, i, j \in[a] \\
\text { distinct }}} C_{1}(h) C_{2}(i) C_{3}(j)+p^{2 k} \sum_{i \neq j \in[a]}\left[\left(p^{k}-p_{2}\right) C_{1} C_{2}(i) C_{3}(j)+\left(p^{k}-p_{3}\right) C_{1} C_{3}(i) C_{2}(j)\right. \\
& \\
&\left.+\left(p^{k}-p_{3}\right) C_{2} C_{3}(i) C_{1}(j)\right]+p^{k}\left(p^{k}-p_{3}\right)\left(p^{k}-p_{3}-p_{2}\right) \sum_{i=1}^{a} C_{1} C_{2} C_{3}(i) .
\end{aligned}
$$

For general $b$, we obtain

$$
\sum_{x \in W\left(l_{1}, \ldots, l_{b}\right)} \phi(\underline{\mathbf{u}})(x)=\sum_{\nu \in \operatorname{Part}[b]} \kappa_{\nu} \cdot \mathcal{C}_{\nu}^{*}
$$

where we define the terms $\kappa_{\nu}$ and $\mathcal{C}_{\nu}^{*}$ as follows. 
Definition 4.14. Let $\nu \in \operatorname{Part}[b]$.

(i) Define $\kappa_{\nu}:=p^{k|\nu|} \cdot \prod_{\tau \in \nu} R_{k}^{\tau}$.

(ii) Let $\nu=\left\{\nu_{1}, \ldots, \nu_{s}\right\}$. Define $\mathcal{C}_{\nu}$ to be the following sum with unrestricted indices (i.e. free to run independently over $1,2, \ldots, a)$

$$
\mathcal{C}_{\nu}:=\sum_{i_{1}, \ldots, i_{s}=1}^{a}\left(\prod_{t \in \nu_{1}} C_{t}\right)\left(i_{1}\right) \cdot\left(\prod_{t \in \nu_{2}} C_{t}\right)\left(i_{2}\right) \cdots\left(\prod_{t \in \nu_{s}} C_{t}\right)\left(i_{s}\right)
$$

In other words, $\mathcal{C}_{\nu}=\prod_{\omega \in \nu}\left(\sum \prod_{t \in \omega} C_{t}\right)$. For example,

$$
\mathcal{C}_{\{\{1,2,4\},\{3,5\},\{6\}\}}=\left(\sum C_{1} C_{2} C_{4}\right)\left(\sum C_{3} C_{5}\right)\left(\sum C_{6}\right) .
$$

(iii) Let $\nu=\left\{\nu_{1}, \ldots, \nu_{s}\right\}$. Define $\mathcal{C}_{\nu}^{*}$ to be the following sum with restricted indices (i.e. satisfying a distinctness condition)

$$
\mathcal{C}_{\nu}^{*}:=\sum_{\substack{i_{1}, \ldots, i_{s} \in[a] \\ \text { distinct }}}\left(\prod_{t \in \nu_{1}} C_{t}\right)\left(i_{1}\right) \cdot\left(\prod_{t \in \nu_{2}} C_{t}\right)\left(i_{2}\right) \cdots\left(\prod_{t \in \nu_{s}} C_{t}\right)\left(i_{s}\right) .
$$

(iv) We define a partial order $\leq$ on Part [b] as follows. For $\nu, \lambda \in \operatorname{Part}[b]$, we say $\nu \leq \lambda$ if $\lambda$ may be obtained from $\nu$ by taking unions of some of the parts of $\nu$. In other words, $\nu$ is a finer partition of $[b]$ than $\lambda$. For example,

$$
\nu=\{\{1,2\},\{3\},\{4\},\{5\},\{6\}\} \leq\{\{1,2,3\},\{4,5,6\}\}=\lambda .
$$

For $\nu \leq \lambda$ and $\tau \in \lambda$, we define $m_{\nu}^{\lambda}(\tau)$ to be the number of parts of $\nu$ which make up $\tau$. In the above example, if $\tau=\{1,2,3\}$ then $m_{\nu}^{\lambda}(\tau)=2$, while if $\tau=\{4,5,6\}$ then $m_{\nu}^{\lambda}(\tau)=3$.

Equation (5) provides an expression for the induced character value $\phi(\underline{\mathbf{u}}) \uparrow_{P_{a p^{k}}}^{\mathfrak{S}_{a p^{k}}}$ on elements of cycle type $p^{l_{1}} \cdots p^{l_{b}}$ in terms of the sums $\mathcal{C}_{\nu}^{*}$ in which the indices are restricted by imposing the distinctness condition. This condition makes equation (5) difficult to apply directly for our target of distinguishing induced characters based on their indexing parameter $\underline{\mathbf{u}}$. It turns out to be much easier to relate the unrestricted sums $\mathcal{C}_{\nu}$ to $\underline{\mathbf{u}}$, and our first objective will be to express $\mathcal{C}_{\nu}^{*}$ in terms of various $\mathcal{C}_{\lambda}$. To that end, it is easy to see that

$$
\mathcal{C}_{\lambda}=\sum_{\nu \geq \lambda} \mathcal{C}_{\nu}^{*}
$$

What we would like is an inverse: to express $\mathcal{C}_{\nu}^{*}$ as a sum of $\mathcal{C}_{\lambda}$ for certain $\lambda$, and then to substitute this into (5). For instance, we may replace the restricted indices $i \neq j \in[a]$ by unrestricted indices in the $\nu=\{\{1,2\},\{3\}\}$ term of (4) by observing that

$$
\sum_{i \neq j \in[a]} C_{1} C_{2}(i) C_{3}(j)=\left(\sum_{i=1}^{a} C_{1} C_{2}(i)\right) \cdot\left(\sum_{j=1}^{a} C_{3}(j)\right)-\sum_{i=1}^{a} C_{1} C_{2} C_{3}(i)=\left(\sum C_{1} C_{2}\right)\left(\sum C_{3}\right)-\left(\sum C_{1} C_{2} C_{3}\right) .
$$

Consider the final two terms above as corresponding to the two members $\omega$ of Part $\nu$, namely $\omega=\{\{\alpha\},\{\beta\}\}$ and $\omega=\{\{\alpha, \beta\}\}$ respectively, where $\nu=\{\alpha, \beta\}, \alpha=\{1,2\}$ and $\beta=\{3\}$. We can perform a similar replacement in general for any number of restricted indices; this is the content of the following lemma.

Lemma 4.15. Let $\nu \in \operatorname{Part}[b]$. Then

$$
\mathcal{C}_{\nu}^{*}=\sum_{\nu \leq \lambda \in \operatorname{Part}[b]}(-1)^{|\nu|-|\lambda|} \prod_{\tau \in \lambda}\left(m_{\nu}^{\lambda}(\tau)-1\right) ! \cdot \mathcal{C}_{\lambda} .
$$


Proof. Let $\mathcal{D}_{\nu}$ be the expression on the right hand side of (6) . We wish to prove that $\mathcal{C}_{\nu}^{*}=\mathcal{D}_{\nu}$.

Suppose $|\nu|=n$. By identifying the members of $\nu$ with $\{1\}, \ldots,\{n\}$, we may without loss of generality assume $\nu=\{\{1\}, \ldots,\{n\}\}$, the minimal element of Part $[n]$ under $\leq$. Since $\mathcal{C}_{\lambda}=$ $\sum_{\alpha \geq \lambda} \mathcal{C}_{\alpha}^{*}$, we have

$$
\mathcal{D}_{\nu}=\sum_{\alpha \in \operatorname{Part}[n]}\left(\sum_{\lambda: \lambda \leq \alpha}(-1)^{n-|\lambda|} \cdot \prod_{\tau \in \lambda}\left(m_{\nu}^{\lambda}(\tau)-1\right) !\right) \mathcal{C}_{\alpha}^{*}
$$

If $\alpha=\nu$, then we obtain the term $1 \cdot \mathcal{C}_{\nu}^{*}$.

Now fix $\alpha \neq \nu$. The coefficient of $\mathcal{C}_{\alpha}^{*}$ in (7) may be rewritten as

$$
\sum_{\lambda: \lambda \leq \alpha}(-1)^{n-|\lambda|} \cdot \prod_{\tau \in \lambda}\left(m_{\nu}^{\lambda}(\tau)-1\right) !=(-1)^{n-2|\lambda|} \cdot \prod_{\tau \in \alpha}\left(\sum_{\rho \in \operatorname{Part} \tau}(-1)^{|\rho|} \prod_{t \in \rho}(|t|-1) !\right) .
$$

For any fixed $\tau \in \alpha$, if $|\tau|=m$ then

$$
\sum_{\rho \in \operatorname{Part} \tau}(-1)^{|\rho|} \prod_{t \in \rho}(|t|-1) !=B_{m}(-0 !,-1 !, \ldots,-(m-1) !) .
$$

Here $B_{m}\left(x_{1}, \ldots, x_{m}\right)$ denotes the $m^{\text {th }}$ complete Bell polynomial: the coefficient of each monomial $\prod_{i} x_{i}^{a_{i}}$ is the number of partitions of $[m]$ such that there are exactly $a_{i}$ members of size $i$, e.g. $B_{4}\left(x_{1}, x_{2}, x_{3}, x_{4}\right)=x_{1}^{4}+6 x_{1}^{2} x_{2}+4 x_{1} x_{3}+3 x_{2}^{2}+x_{4}$. Moreover, by the determinantal form $B_{m}\left(x_{1}, \ldots, x_{m}\right)=\operatorname{det} \mathrm{B}$ where $\mathrm{B}$ is the $m \times m$ matrix with

$$
\mathrm{B}_{i, i+j}=\frac{x_{j+1}}{j !} \forall j \geq 0, \quad \mathrm{~B}_{i, i-1}=-i+1 \quad \text { and } \quad \mathrm{B}_{i, j}=0 \text { otherwise, }
$$

it is clear that if $m \geq 2$ then $B_{m}\left(x_{1}, \ldots, x_{m}\right)=0$ when $x_{i}=-(i-1)$ ! for all $i$. Since $\alpha \neq \nu$, there exists some $\tau \in \alpha$ with $|\tau| \geq 2$. The assertion of the lemma then follows from (17).

Corollary 4.16. We have that

where

$$
\sum_{x \in W\left(l_{1}, \ldots, l_{b}\right)} \phi(\underline{\mathbf{u}})(x)=\sum_{\lambda \in \operatorname{Part}[b]} \theta_{\lambda} \cdot \mathcal{C}_{\lambda},
$$

$$
\theta_{\lambda}:=\sum_{\nu \leq \lambda} p^{k|\nu|} \cdot \prod_{\tau \in \nu} R_{k}^{\tau} \cdot(-1)^{|\nu|-|\lambda|} \prod_{z \in \lambda}\left(m_{\nu}^{\lambda}(\tau)-1\right) ! .
$$

Proof. This follows from Definition 4.14 and substituting Lemma 4.15 into (5).

Comparing with the assertion of Theorem 4.13 , it therefore remains to prove that

$$
\theta_{\lambda}=(-1)^{b-|\lambda|} \cdot p^{k|\lambda|} \cdot \prod_{\tau \in \lambda} M(\tau) .
$$

To show that (8) holds, we view $\theta_{\lambda}$ as a polynomial in the term $p^{k}$ and evaluate the coefficients as follows. Fix $\lambda \in \operatorname{Part}[b]$. For $u \geq|\lambda|$, we define the quantities $T_{u}$ via:

$$
\theta_{\lambda}=: \sum_{u \geq|\lambda|} p^{u k} \cdot T_{u}
$$


For example, let $b=3$ and $\lambda=\{\{1,2,3\}\}$. Then the set of those $\nu \leq \lambda$ is all of Part [3], and so

$$
\begin{aligned}
& \theta_{\lambda} \quad=\sum_{\nu \leq \lambda} p^{k|\nu|} \cdot \prod_{\tau \in \nu} R_{k}^{\tau} \cdot(-1)^{|\nu|-|\lambda|} \prod_{z \in \lambda}\left(m_{\nu}^{\lambda}(\tau)-1\right) \text { ! } \\
& =p^{k}\left(p^{k}-p_{3}\right)\left(p^{k}-p_{3}-p_{2}\right) \\
& -p^{2 k}\left(p^{k}-p_{2}\right) \\
& -p^{2 k}\left(p^{k}-p_{3}\right) \\
& -p^{2 k}\left(p^{k}-p_{3}\right) \\
& +2 p^{3 k} \\
& \nu=\{\{1,2,3\}\} \\
& \nu=\{\{1,2\},\{3\}\} \\
& \nu=\{\{1,3\},\{2\}\} \\
& \nu=\{\{2,3\},\{1\}\} \\
& \nu=\{\{1\},\{2\},\{3\}\} \\
& \Longrightarrow \quad T_{1}=p_{3}\left(p_{3}+p_{2}\right), \quad T_{2}=\left(-2 p_{3}-p_{2}\right)+p_{2}+p_{3}+p_{3}, \\
& T_{3}=1-1-1-1+2 \text {. }
\end{aligned}
$$

This example and (8) motivate the following lemma.

Lemma 4.17. Fix $\lambda \in \operatorname{Part}[b]$. Then $T_{|\lambda|}=(-1)^{b-|\lambda|} \prod_{\tau \in \lambda} M(\tau)$ and $T_{u}=0$ for all $u>|\lambda|$.

In order to prove Lemma 4.17, we introduce some definitions.

Definition 4.18. Let $n \in \mathbb{N}, \alpha \in \operatorname{Part}[n]$ and $i \in \mathbb{N}_{0}$.

(i) Define $\mathcal{M}_{i}(\alpha)$ to be the coefficient of $p^{i k}$ in $\prod_{\tau \in \alpha} R_{k}^{\tau}$ (see Definition 4.8), viewed as a polynomial in $p^{k}$. In particular, $\mathcal{M}_{0}(\alpha)=\prod_{\tau \in \alpha}(-1)^{|\tau|-1} M(\tau)=(-1)^{n-|\alpha|} \prod_{\tau \in \alpha} M(\tau)$ (see Definition 4.12 for $M(\tau)$ ).

For example, when $\alpha=\{\{1,2\},\{3,4,5\}\} \in \operatorname{Part}[5], \mathcal{M}_{0}(\alpha)=-p_{2} p_{5}\left(p_{5}+p_{4}\right)$ which is the coefficient of $p^{0 \cdot k}$ in $\left(p^{k}-p_{2}\right)\left(p^{k}-p_{5}\right)\left(p^{k}-p_{5}-p_{4}\right)$.

(ii) Define $\operatorname{Cut}(\alpha, i)=\{\beta \in \operatorname{Part}[n]: \beta \leq \alpha$ and $|\beta|=|\alpha|+i\}$. In other words, we may think of $\beta$ as being obtained from $\alpha$ by cutting $i$ times, e.g.

$$
\alpha=\{\{1,2\},\{3,4,5\}\}, \quad \beta=\{\{1\},\{2\},\{4\},\{3,5\}\} \in \operatorname{Cut}(\alpha, 2) .
$$

In particular, $\operatorname{Cut}(\{[n]\}, i)=\{\beta \in \operatorname{Part}[n]:|\beta|=1+i\}$.

Remark 4.19. We have that

$$
\mathcal{M}_{0}(\{[n]\})=(-1)^{n-1} M([n])=(-1)^{n-1} \prod_{a=2}^{n}\left(p_{n}+p_{n-1}+\cdots+p_{a}\right)
$$

Similarly, the coefficient $\mathcal{M}_{i}(\{[n]\})$ is equal to $(-1)^{n-1-i}$ times the sum of all products of $n-1-i$ of the terms in the product on the right hand side of (9). Since the term $p_{n}$ appears in every bracket, we may express this sum as a partial derivative with respect to $p_{n}$ in the following way. Treat $M([n])$ as a polynomial in indeterminates $p_{j}$, and treat $\mathcal{M}_{i}(\{[n]\})$ similarly. Then

$$
\mathcal{M}_{i}(\{[n]\})=-\frac{1}{i} \frac{\partial}{\partial p_{n}} \mathcal{M}_{i-1}(\{[n]\}),
$$

since each choice of $n-1-i$ terms from the product on the right hand side of (9) can be obtained in $i$ different ways by first choosing $n-i$ terms and then discarding one of them.

Lemma 4.20. Let $n \in \mathbb{N}$ and $i \in \mathbb{N}_{0}$. Then

$$
\mathcal{M}_{i}(\{[n]\})=\sum_{\mu \in \operatorname{Cut}(\{[n]\}, i)} \mathcal{M}_{0}(\mu)
$$

Proof. Since the maximal power of $p^{k}$ in $R_{k}^{[n]}$ is $p^{(n-1) k}$, the statement is true when $i \geq n$ as $\operatorname{Cut}(\{[n]\}, i)=\emptyset$. Moreover, the cases $n \leq 2$ and $i=0$ are trivial, so we may now assume $n \geq 3$ and $i \in[n-1]$. 
We proceed by induction on $i$ : first suppose $i=1$. Let $a \in\{2,3, \ldots, n\}$ and write $J_{a}$ for the expression obtained by dropping the factor $\left(p_{n}+\ldots+p_{a}\right)$ from the product $M([n])$. That is,

$$
\begin{aligned}
J_{a}: & =\frac{M([n])}{p_{n}+p_{n-1}+\ldots+p_{a}} \\
& =p_{n} \cdots\left(p_{n}+p_{n-1}+\cdots+p_{a+1}\right)\left(p_{n}+p_{n-1}+\cdots+p_{a-1}\right) \cdots\left(p_{n}+p_{n-1}+\cdots+p_{2}\right) .
\end{aligned}
$$

Recalling Remark 4.19, we have that $\mathcal{M}_{1}(\{[n]\})=\sum_{a=2}^{n}(-1)^{n-2} J_{a}$. We claim that

$$
J_{a}=\Xi_{a}
$$

where we define

$$
\Xi_{a}:=\sum_{Y \subseteq[a-2]} M(\{Y \cup\{a-1\}\}) \cdot M(\{[n] \backslash(Y \cup\{a-1\})\})
$$

with $[0]:=\emptyset$. The assertion of the present lemma for $i=1$ then follows by summing $J_{a}=\Xi_{a}$ over $a \in\{2,3, \ldots, n\}$, since $\Xi_{a}$ is precisely $(-1)^{n-2} \mathcal{M}_{0}(\mu)$ summed over those $\mu \in \operatorname{Cut}(\{[n]\}, 1)$ such that, writing $\mu=\left\{\mu_{1}, \mu_{2}\right\}$ where $n \in \mu_{2}$, we have $\max \left(\mu_{1}\right)=a-1$.

We prove $J_{a}=\Xi_{a}$ by induction on $a$. Consider first the base case $a=2$. In this case, observe $J_{2}=p_{n}\left(p_{n}+p_{n-1}\right) \cdots\left(p_{n}+p_{n-1}+\cdots+p_{3}\right)$ by definition, while

$$
\Xi_{2}=M(\{1\}) \cdot M(\{[n] \backslash\{1\}\})=1 \cdot p_{n}\left(p_{n}+p_{n-1}\right) \cdots\left(p_{n}+p_{n-1}+\cdots+p_{3}\right) .
$$

For $a>2$, we partition the sum over $Y \subseteq[a-2]$ in $\Xi_{a}$ according to whether the element $a-2$ belongs to $Y$, so that $\Xi_{a}=\Xi_{a, 1}+\Xi_{a, 2}$ where

$$
\Xi_{a, 1}=\sum_{Y \subseteq[a-3]} M(\{Y \cup\{a-1\}\}) \cdot M(\{[n] \backslash(Y \cup\{a-1\})\})
$$

and

$$
\Xi_{a, 2}=\sum_{Y \subseteq[a-3]} M(\{Y \cup\{a-1, a-2\}\}) \cdot M(\{[n] \backslash(Y \cup\{a-1, a-2\})\}) .
$$

Observe now that replacing all instances of $p_{a-1}$ by $p_{a-2}$ and vice versa in the expression for $\Xi_{a, 1}$ yields $\Xi_{a-1}$. But the inductive hypothesis implies that $\Xi_{a-1}=J_{a-1}$, and we note that in the product

$J_{a-1}=p_{n}\left(p_{n}+p_{n-1}\right) \cdots\left(p_{n}+p_{n-1}+\cdots+p_{a}\right)\left(p_{n}+p_{n-1}+\cdots+p_{a-2}\right) \cdots\left(p_{n}+p_{n-1}+\cdots+p_{2}\right)$ the terms $p_{a-1}$ and $p_{a-2}$ appear in the same brackets. Therefore swapping $p_{a-1}$ and $p_{a-2}$ in the expression for $J_{a-1}$ leaves it unchanged, and so $\Xi_{a, 1}=J_{a-1}$.

Next, observe that $M(\{Y \cup\{a-1, a-2\}\})$ is equal to $p_{a-1}$ times the expression obtained by replacing all occurrences of $p_{a-2}$ with $\left(p_{a-1}+p_{a-2}\right)$ in $M(\{Y \cup\{a-2\}\})$ - this is easily seen from Definition 4.12. Moreover, by our induction hypothesis we have that

$$
\sum_{Y \subseteq[a-3]} M(\{Y \cup\{a-2\}\}) \cdot M(\{[n] \backslash(Y \cup\{a-1, a-2\})\})=\frac{M([n] \backslash\{a-1\})}{p_{n}+p_{n-1}+\ldots+p_{a}} .
$$

Replacing all occurrences of $p_{a-2}$ with $\left(p_{a-1}+p_{a-2}\right)$ on the right hand side of (10) yields

$$
\frac{M([n])}{\left(p_{n}+p_{n-1}+\ldots+p_{a}\right)\left(p_{n}+p_{n-1}+\ldots+p_{a}+p_{a-1}\right)}=\frac{J_{a-1}}{p_{n}+p_{n-1}+\ldots+p_{a}}
$$

and so

$$
\Xi_{a, 2}=p_{a-1} \frac{J_{a-1}}{p_{n}+p_{n-1}+\ldots+p_{a}}=J_{a}-J_{a-1}
$$


So $\Xi_{a}=\Xi_{a, 1}+\Xi_{a, 2}=J_{a}$ as required. This proves $J_{a}=\Xi_{a}$ for all $a \in\{2,3, \ldots, n\}$, and hence the case of $i=1$ is concluded.

Now suppose $i>1$. As in Remark4.19, we may express $\mathcal{M}_{i}(\{[n]\})$ in terms of $\frac{\partial}{\partial p_{n}} \mathcal{M}_{i-1}(\{[n]\})$, and using the induction hypothesis we obtain

$$
\begin{aligned}
\mathcal{M}_{i}(\{[n]\}) & =-\frac{1}{i} \frac{\partial}{\partial p_{n}} \mathcal{M}_{i-1}(\{[n]\})=-\frac{1}{i} \sum_{\mu \in \operatorname{Cut}(\{[n]\}, i-1)} \frac{\partial}{\partial p_{n}} \mathcal{M}_{0}(\mu) \\
& =\frac{1}{i} \sum_{\mu \in \operatorname{Cut}(\{[n]\}, i-1)} \mathcal{M}_{1}\left(\left\{\mu_{1}\right\}\right) \cdot \mathcal{M}_{0}\left(\left\{\mu_{2}\right\}\right) \cdots \mathcal{M}_{0}\left(\left\{\mu_{i}\right\}\right)
\end{aligned}
$$

where $\mu:=\left\{\mu_{1}, \ldots, \mu_{i}\right\}$ and $n \in \mu_{1}$. By using the inductive hypothesis on $\mathcal{M}_{1}\left(\left\{\mu_{1}\right\}\right)$, we obtain

$$
\begin{aligned}
\mathcal{M}_{i}(\{[n]\}) & =\frac{1}{i} \sum_{\mu \in \operatorname{Cut}(\{[n]\}, i-1)} \sum_{\substack{\nu=\left\{\nu_{1}, \nu_{2}\right\} \\
\in \operatorname{Cut}\left(\left\{\mu_{1}\right\}, 1\right)}} \mathcal{M}_{0}\left(\left\{\nu_{1}\right\}\right) \cdot \mathcal{M}_{0}\left(\left\{\nu_{2}\right\}\right) \cdot \prod_{j=2}^{i} \mathcal{M}_{0}\left(\left\{\mu_{j}\right\}\right) \\
& =\frac{1}{i} \sum_{\gamma \in \operatorname{Cut}(\{[n]\}, i)} N_{\gamma} \cdot \mathcal{M}_{0}(\gamma)
\end{aligned}
$$

where $N_{\gamma}$ is the number of ways of obtaining $\gamma \in \operatorname{Cut}(\{[n]\}, i)$ by first taking some $\mu \in$ $\operatorname{Cut}(\{[n]\}, i-1)$ then replacing the part $\mu_{1}$ of $\mu$ containing the number $n$ by $\nu_{1}, \nu_{2}$, where $\left\{\nu_{1}, \nu_{2}\right\} \in \operatorname{Cut}\left(\left\{\mu_{1}\right\}, 1\right)$. But for all $\gamma$, we have $N_{\gamma}=i$ since $|\gamma|=i+1$ and $\mu$ is uniquely determined once one of $\{\tau \in \gamma: n \notin \tau\}$ is chosen to be $\nu_{2}$ (assuming without loss of generality that $n \in \nu_{1}$ ). This concludes the proof.

Corollary 4.21. Let $n \in \mathbb{N}$ and $i \in \mathbb{N}_{0}$. Let $\alpha \in \operatorname{Part}[n]$. Then

$$
\mathcal{M}_{i}(\alpha)=\sum_{\beta \in \operatorname{Cut}(\alpha, i)} \mathcal{M}_{0}(\beta) .
$$

Proof. We proceed by induction on $|\alpha|$, with Lemma 4.20 giving the base case $|\alpha|=1$. In particular, we may now assume $|\alpha| \geq 2$. Fix some $\tau \in \alpha$. Then

$$
\begin{aligned}
\sum_{\beta \in \operatorname{Cut}(\alpha, i)} \mathcal{M}_{0}(\beta) & =\sum_{j \geq 0} \sum_{\substack{\beta \in \operatorname{Cut}(\alpha, i) \text { where } \tau \text { is } \\
\text { cut exactly j times }}} \mathcal{M}_{0}(\beta)=\sum_{j \geq 0} \sum_{\substack{\gamma \in \operatorname{Cut}(\{\tau\}, j), \delta \in \operatorname{Cut}(\alpha \backslash\{\tau\}, i-j)}} \mathcal{M}_{0}(\gamma) \cdot \mathcal{M}_{0}(\delta) \\
& =\sum_{j \geq 0} \mathcal{M}_{j}(\{\tau\}) \cdot \mathcal{M}_{i-j}(\alpha \backslash\{\tau\})=\mathcal{M}_{i}(\alpha)
\end{aligned}
$$

where the third equality follows from Lemma 4.20 and the inductive hypothesis.

Proof of Lemma 4.17. The value of $T_{|\lambda|}$ follows immediately from Corollary 4.16, so from now on assume $u>|\lambda|$. By Corollary 4.16 and Definition 4.18, the coefficient in $\theta_{\lambda}$ of $p^{u k}$ is

$$
\begin{aligned}
T_{u} & =\sum_{\substack{\nu \leq \lambda \\
|\nu| \leq u}} \mathcal{M}_{u-|\nu|}(\nu) \cdot(-1)^{|\nu|-|\lambda|} \prod_{\tau \in \lambda}\left(m_{\nu}^{\lambda}(\tau)-1\right) ! \\
& =\sum_{\substack{\nu \leq \lambda \\
|\nu| \leq u}} \sum_{\beta \in \operatorname{Cut}(\nu, u-|\nu|)} \mathcal{M}_{0}(\beta) \cdot(-1)^{|\nu|-|\lambda|} \prod_{\tau \in \lambda}\left(m_{\nu}^{\lambda}(\tau)-1\right) ! \\
& =\sum_{\substack{\beta \leq \lambda \\
|\beta|=u}} \mathcal{M}_{0}(\beta) \cdot \sum_{\nu: \beta \leq \nu \leq \lambda}(-1)^{|\nu|-|\lambda|} \prod_{\tau \in \lambda}\left(m_{\nu}^{\lambda}(\tau)-1\right) !
\end{aligned}
$$




$$
=\sum_{\substack{\beta \leq \lambda \\|\beta|=u}} \mathcal{M}_{0}(\beta) \cdot \prod_{\tau \in \lambda} \sum_{\sigma \in \operatorname{Part}\left[m_{\beta}^{\lambda}(\tau)\right]}(-1)^{|\sigma|-1}(|\sigma|-1) !
$$

where the second equality follows from Corollary 4.21. From the following well-known identity involving Stirling numbers of the second kind $S(n, j)$ and the polylogarithm Li,

$$
\sum_{j=1}^{n} S(n, j) \cdot(j-1) ! w^{j}=(-1)^{n} \operatorname{Li}_{1-n}\left(1+\frac{1}{w}\right),
$$

we obtain

$$
\sum_{\sigma \in \operatorname{Part}[n]}(-1)^{|\sigma|}(|\sigma|-1) !=0
$$

for all $n \in \mathbb{N}$. Substituting this into (11) with $n=m_{\beta}^{\lambda}(\tau)$, we obtain $T_{u}=0$ as desired.

Since (8) follows immediately from Lemma4.17, the proof of Theorem 4.13 is finally concluded.

\section{Proof of Theorem 3.6}

Theorem 4.13 gives us a formula with which we can evaluate $\phi(\underline{\mathbf{s}}) \uparrow^{\mathfrak{S}_{a p^{k}}}$ on elements of certain cycle types. The strategy for the proof of Theorem 3.6 is as follows.

We first use Theorem 4.13 to translate the equality of characters

$$
\phi(\underline{\mathbf{s}}) \uparrow^{\mathfrak{S}_{a p^{k}}}=\phi(\underline{\mathbf{t}}) \uparrow^{\mathfrak{S}_{a p^{k}}}
$$

into a collection of equations relating the parameters $\underline{\mathbf{s}}$ and $\underline{\mathbf{t}}$ (see Corollary 5.1 ). These equations, however, are in terms of the functions $C$ described in Definition 4.3, and it remains to relate these functions to the entries of the sequences in $\underline{\mathbf{s}}$ and $\underline{\mathbf{t}}$ themselves. This will be achieved in Proposition 5.4 following two technical lemmas.

Proposition [5.4 tells us that if $\phi(\underline{\mathbf{s}}) \uparrow^{\mathfrak{S}_{a p^{k}}}=\phi(\underline{\mathbf{t}}) \uparrow^{\mathfrak{S}_{a p^{k}}}$ then we have equality between multisets of values obtained from certain partial sums of entries of $\underline{\mathbf{s}}$ and $\underline{\mathbf{t}}$. It still remains to show these equalities of partial sums provide enough information to conclude that Theorem 3.6 holds. This will follow from a combinatorial argument encapsulated within Lemma 5.7 and Theorem 5.8 .

Corollary 5.1. Let $a \in[p-1]$. Let $\phi(\underline{\mathbf{s}}), \phi(\underline{\mathbf{t}}) \in \operatorname{Lin}\left(P_{a p^{k}}\right)$. Suppose that $\phi(\underline{\mathbf{s}}) \uparrow^{\mathfrak{S}_{a p^{k}}}=\phi(\underline{\mathbf{t}}) \uparrow^{\mathfrak{S}_{a p^{k}}}$. Let $b \in[a]$ and suppose $l_{1}, l_{2}, \ldots, l_{b}$ are integers such that $1 \leq l_{1}<\cdots<l_{b} \leq k$. Then

$$
\sum_{j=1}^{a} C_{l_{1}}\left(\mathrm{~s}^{j}\right) \cdot C_{l_{2}}\left(\mathrm{~s}^{j}\right) \cdots C_{l_{b}}\left(\mathrm{~s}^{j}\right)=\sum_{j=1}^{a} C_{l_{1}}\left(\mathrm{t}^{j}\right) \cdot C_{l_{2}}\left(\mathrm{t}^{j}\right) \cdots C_{l_{b}}\left(\mathrm{t}^{j}\right) .
$$

Proof. We proceed by induction on $b$. First suppose $b=1$ and let $g$ be an element of $\mathfrak{S}_{a p^{k}}$ of cycle type $p^{l_{1}}$. By Theorem 4.13 ,

$$
\phi(\underline{\mathbf{s}}) \uparrow^{\mathfrak{S}_{a p^{k}}}(g)=\frac{\left|\mathbf{C}_{\mathfrak{S}_{a p^{k}}}(g)\right|}{\left|P_{a p^{k}}\right|} \cdot \sum_{x \in W\left(l_{1}\right)} \phi(\underline{\mathbf{s}})(x)=\frac{\left|\mathbf{C}_{\mathfrak{S}_{a p^{k}}}(g)\right|}{\left|P_{a p^{k}}\right|} \cdot p^{k} \cdot \sum_{i=1}^{a} C_{l_{1}}\left(\mathrm{~s}^{i}\right),
$$

and similarly for $\underline{\mathbf{t}}$. Since $\phi(\underline{\mathbf{s}}) \uparrow^{\mathfrak{S}_{a p^{k}}}=\phi(\underline{\mathbf{t}}) \uparrow^{\mathfrak{S}_{a p^{k}}}$, the assertion follows.

Now suppose $b \geq 2$. Let $g \in \mathfrak{S}_{a p^{k}}$ of cycle type $p^{l_{1}} \cdots p^{l_{b}}$. By Theorem 4.13,

$$
\phi(\underline{\mathbf{s}}) \uparrow^{\mathfrak{S}_{a p^{k}}}(g)=\frac{\left|\mathbf{C}_{\mathfrak{S}_{a p^{k}}}(g)\right|}{\left|P_{a p^{k}}\right|} \cdot \sum_{\nu \in \operatorname{Part}[b]}(-1)^{b-|\nu|} p^{k|\nu|} \prod_{\tau \in \nu}\left[M(\tau) \cdot \sum_{i=1}^{a}\left(\prod_{z \in \tau} C_{l_{z}}\left(\mathrm{~s}^{i}\right)\right)\right] .
$$


By the inductive hypothesis, $\sum_{i=1}^{a}\left(\prod_{z \in \tau} C_{l_{z}}\left(\mathrm{~s}^{i}\right)\right)=\sum_{i=1}^{a}\left(\prod_{z \in \tau} C_{l_{z}}\left(\mathrm{t}^{i}\right)\right)$ for every $\tau$ satisfying $|\tau|<b$. The only $\tau$ such that $|\tau|=b$ comes from $\nu=\{[b]\}$ (giving $\tau=[b]$ ). Using $\phi(\underline{\mathbf{s}}) \uparrow^{\mathfrak{S}_{a p^{k}}}=$ $\phi(\underline{\mathbf{t}})^{\mathfrak{S}_{a p^{k}}}$ and substituting these observations into (12), we obtain

$$
(-1)^{b-1} p^{k} \cdot M([b]) \cdot \sum_{i=1}^{a} C_{l_{1}}\left(\mathrm{~s}^{i}\right) \cdots C_{l_{b}}\left(\mathrm{~s}^{i}\right)=(-1)^{b-1} p^{k} \cdot M([b]) \cdot \sum_{i=1}^{a} C_{l_{1}}\left(\mathrm{t}^{i}\right) \cdots C_{l_{b}}\left(\mathrm{t}^{i}\right) .
$$

Since $(-1)^{b-1} p^{k} \cdot M([b]) \neq 0$, the assertion follows.

From Corollary 5.1 we would like to deduce Proposition 5.4 which states, informally, that certain partial sums of the sequences $\mathrm{s}^{1}, \ldots, \mathrm{s}^{a}$ and $\mathrm{t}^{1}, \ldots, \mathrm{t}^{a}$ are equal. Recall from Definition 4.3 that the part of $C_{l}(\mathrm{u})$ depending on $\mathrm{u}$ is

$$
\prod_{m=1}^{l}\left(p \mathbf{u}_{m}-1\right)=(-1)^{l} \cdot(1-p)^{\sum_{m=1}^{l} \mathbf{u}_{m}} .
$$

Corollary 5.1 thus implies equality between certain sums of powers of $1-p$. The following technical lemma will be used (with $q=p-1$ ) to help translate these equalities into more directly applicable information.

For notational convenience, we denote multisets by asterisks.

Lemma 5.2. Let $q \in \mathbb{N}_{\geq 2}$. Let $a \in[q]$ and let $\sigma_{j}, \tau_{j} \in \mathbb{N}_{0}$ for $j \in[a]$. If

$$
\sum_{j=1}^{a}(-q)^{\sigma_{j}}=\sum_{j=1}^{a}(-q)^{\tau_{j}}
$$

then either

(i) $\left\{\sigma_{1}, \ldots, \sigma_{a}\right\}^{*}=\left\{\tau_{1}, \ldots, \tau_{a}\right\}^{*}$ is an equality of multisets; or

(ii) $a=q$ and the multisets $\left\{\sigma_{1}, \ldots, \sigma_{a}\right\}^{*}$ and $\left\{\tau_{1}, \ldots, \tau_{a}\right\}^{*}$ are $\{w, w-1, \ldots, w-1\}^{*}$ and $\{w-2, \ldots, w-2\}^{*}$ for some $w \in \mathbb{N}_{\geq 2}$.

Proof. We proceed by induction on $a$. The assertion is clear if $a=1$, so now assume $2 \leq a \leq q$, and suppose $\left\{\sigma_{1}, \ldots, \sigma_{a}\right\}^{*} \neq\left\{\tau_{1}, \ldots, \tau_{a}\right\}^{*}$. If $\sigma_{i}=\tau_{j}$ for some $i, j \in[a]$, then by the inductive hypothesis for $a-1 \neq q$, we have $\left\{\sigma_{1}, \ldots, \sigma_{i-1}, \sigma_{i+1}, \ldots, \sigma_{a}\right\}^{*}=\left\{\tau_{1}, \ldots, \tau_{j-1}, \tau_{j+1}, \ldots, \tau_{a}\right\}^{*}$. But then $\left\{\sigma_{1}, \ldots, \sigma_{a}\right\}^{*}=\left\{\tau_{1}, \ldots, \tau_{a}\right\}^{*}$, a contradiction. Thus $\sigma_{i} \neq \tau_{j}$ for all $i, j \in[a]$. Without loss of generality suppose $\sigma_{1}=\max \left\{\sigma_{i}, \tau_{j}\right\}_{i, j \in[a]}$, so in particular $\tau_{j}<\sigma_{1}$ for all $j$. By multiplying by $-q$ if necessary (equivalently, by adding 1 to all $\sigma_{i}$ and $\tau_{j}$ ), we may further assume that $\sigma_{1}$ is even. Then

$$
q^{\sigma_{1}-1} \leq q^{\sigma_{1}}-(a-1) q^{\sigma_{1}-1} \leq \sum_{j=1}^{a} q^{\sigma_{j}}(-1)^{\sigma_{j}}=\sum_{j=1}^{a} q^{\tau_{j}}(-1)^{\tau_{j}} \leq a q^{\sigma_{1}-2} \leq q^{\sigma_{1}-1} .
$$

Hence all inequalities in the above must hold with equalities, implying that $a=q, \sigma_{j}=\sigma_{1}-1$ for all $j \neq 1$, and $\tau_{j}=\sigma_{1}-2$ for all $j \in[a]$. This is exactly case (ii).

Unfortunately, the existence of case (ii) of Lemma 5.2 creates a modest technical hurdle. In order to rule out this case in our application of Lemma 5.2 to the equations from Corollary 5.1, we will provide one additional equation which will imply, informally, that the sequences $\mathrm{s}^{1}, \ldots, \mathrm{s}^{a}$ and $t^{1}, \ldots, t^{a}$ have equal partial sums. This is the content of Lemma 5.3 below. In order to provide this additional equation, we use one more explicit computation of induced character values, in a case not covered by Theorem 4.13, the case where $g$ is the product of two disjoint $p^{l}$-cycles. 
This computation follows very similar lines to the calculations in Section 4. This case is no more difficult because when $n=p^{k}, p \geq 3$ and $g$ is a product of two $p^{l}$-cycles, then $g$ has a fixed point in $[n]$ and the inductive argument will avoid working with the wreath product structure as before (see Remark 4.1). The assumption in the following Lemma that $p$ is an odd prime is therefore crucial, since when $p=2$ the situation is complicated by the fact that a product of two disjoint cycles of length $2^{k-1}$ in $\mathfrak{S}_{2^{k}}$ has no fixed point.

Lemma 5.3. Let $p$ be an odd prime, $a \in\{2,3, \ldots, p-1\}, k \in \mathbb{N}$ and $l \in[k]$. Let $\phi(\underline{\mathbf{s}})$, $\phi(\underline{\mathbf{t}}) \in \operatorname{Lin}\left(P_{a p^{k}}\right)$ and suppose $\phi(\underline{\mathbf{s}}) \uparrow^{\mathfrak{S}_{a p^{k}}}=\phi(\underline{\mathbf{t}}) \uparrow^{\mathfrak{S}_{a p^{k}}}$. Then

$$
\left\{\sum_{m=1}^{l} \mathrm{~s}_{m}^{1}, \ldots, \sum_{m=1}^{l} \mathrm{~s}_{m}^{a}\right\}^{*}=\left\{\sum_{m=1}^{l} \mathrm{t}_{m}^{1}, \ldots, \sum_{m=1}^{l} \mathrm{t}_{m}^{a}\right\}^{*} .
$$

Proof. Let $\mathrm{u} \in\{0,1\}^{k}$ and let $\mathrm{u}^{-}=\left(\mathrm{u}_{1}, \ldots, \mathrm{u}_{k-1}\right)$. First suppose $l<k$. Recall the set $X(l, l)$ from Definition [4.3, and $\Gamma_{l, l ; k}(\mathrm{u})$ from Definition 4.2. Consider some $x \in X(l, l)$. Since $x$ must have a fixed point, $x=\left(f_{1}, \ldots, f_{p} ; 1\right)$ with $f_{1}, \ldots, f_{p} \in P_{p^{k-1}}$, and either $f_{i}, f_{j} \in Y(l)$ for some distinct $i, j \in[p]$ and $f_{h}=1$ for all $h \notin\{i, j\}$, or $f_{i} \in Y(l, l)$ for a unique $i \in[p]$ and $f_{j}=1$ for all $j \neq i$. Thus by Lemma 3.1.

$$
\Gamma_{l, l ; k}(\mathbf{u})=\left(\begin{array}{l}
p \\
2
\end{array}\right) \cdot \Gamma_{l ; k-1}\left(\mathbf{u}^{-}\right) \cdot \Gamma_{l ; k-1}\left(\mathbf{u}^{-}\right)+p \cdot \Gamma_{l, l ; k-1}\left(\mathbf{u}^{-}\right) .
$$

Iterating (13) and recalling that $\Gamma_{l, l ; l}(-)=0$, we obtain

$$
\Gamma_{l, l ; k}(\mathbf{u})=\left(\begin{array}{c}
p \\
2
\end{array}\right) \sum_{i=l}^{k-1} p^{k-1-i} \cdot \Gamma_{l ; i}\left(\left(\mathbf{u}_{1}, \ldots, \mathbf{u}_{i}\right)\right)^{2}=\left(\begin{array}{c}
p \\
2
\end{array}\right) \sum_{i=l}^{k-1} p^{k-1+i} \cdot C_{l}(\mathbf{u})^{2}=\frac{p^{k}\left(p^{k}-p^{l}\right)}{2} \cdot C_{l}(\mathbf{u})^{2}
$$

where the second equality follows from Lemma 4.4.

Next, let $\phi(\underline{\mathbf{u}}) \in \operatorname{Lin}\left(P_{a p^{k}}\right)$. We consider the value of $\phi(\underline{\mathbf{u}}) \uparrow^{\mathfrak{S}_{a p^{k}}}$ on elements of cycle type $p^{l} p^{l}$. Let $g \in \mathfrak{S}_{a p^{k}}$ have cycle type $p^{l} p^{l}$. Recall the set $W(l, l)$ from Definition 4.10, Then

$$
\phi(\underline{\mathbf{u}}) \uparrow_{P_{a p^{k}}}^{\mathfrak{S}_{a p^{k}}}(g)=\frac{\mid \mathbf{C}_{\mathfrak{G}_{a p^{k}}(g) \mid}}{\left|P_{a p^{k}}\right|} \cdot \sum_{x \in W(l, l)} \phi(\underline{\mathbf{u}})(x) .
$$

Consider some $x \in W(l, l)$. Since $P_{a p^{k}} \cong P_{p^{k}} \times \cdots \times P_{p^{k}}$ ( $a$ times), we can write $x=x_{1} \cdots x_{a}$ with each $x_{i}$ in a distinct direct factor $P_{p^{k}}$. Moreover, either $x_{i}, x_{j} \in X(l)$ for some distinct $i, j \in[a]$ and $x_{h}=1$ for all $h \notin\{i, j\}$, or $x_{i} \in X(l, l)$ for a unique $i \in[a]$ and $x_{j}=1$ for all $j \neq i$. Hence

$$
\begin{aligned}
\sum_{x \in W(l, l)} \phi(\underline{\mathbf{u}})(x) & =\sum_{\{i, j\} \subseteq[a]} \sum_{x_{i}, x_{j} \in X(l)} \phi\left(\mathbf{u}^{i}\right)\left(x_{i}\right) \cdot \phi\left(\mathbf{u}^{j}\right)\left(x_{j}\right)+\sum_{i=1}^{a} \sum_{x_{i} \in X(l, l)} \phi\left(\mathbf{u}^{i}\right)\left(x_{i}\right) \\
& =\sum_{1 \leq i<j \leq a} \Gamma_{l ; k}\left(\mathbf{u}^{i}\right) \cdot \Gamma_{l ; k}\left(\mathbf{u}^{j}\right)+\sum_{i=1}^{a} \Gamma_{l, l ; k}\left(\mathbf{u}^{i}\right) \\
& =\sum_{1 \leq i<j \leq a} p^{k} C_{l}\left(\mathbf{u}^{i}\right) \cdot p^{k} C_{l}\left(\mathbf{u}^{j}\right)+\sum_{i=1}^{a} \frac{p^{k}\left(p^{k}-p^{l}\right)}{2} \cdot C_{l}\left(\mathbf{u}^{\mathrm{i}}\right)^{2} \\
& =\frac{p^{2 k}}{2}\left[\left(\sum_{i=1}^{a} C_{l}\left(\mathbf{u}^{i}\right)\right) \cdot\left(\sum_{j=1}^{a} C_{l}\left(\mathbf{u}^{j}\right)\right)-\sum_{i=1}^{a} C_{l}\left(\mathbf{u}^{i}\right)^{2}\right]+\frac{p^{k}\left(p^{k}-p^{l}\right)}{2} \cdot \sum_{i=1}^{a} C_{l}\left(\mathbf{u}^{i}\right)^{2}
\end{aligned}
$$




$$
=\frac{p^{2 k}}{2}\left(\sum_{i=1}^{a} C_{l}\left(\mathbf{u}^{i}\right)\right)\left(\sum_{j=1}^{a} C_{l}\left(\mathbf{u}^{j}\right)\right)-\frac{p^{k} \cdot p^{l}}{2} \cdot \sum_{i=1}^{a} C_{l}\left(\mathbf{u}^{i}\right)^{2} .
$$

Since $\phi(\underline{\mathbf{s}})^{\uparrow} \mathfrak{S}_{a p^{k}}\left(g^{\prime}\right)=\phi(\underline{\mathbf{t}}) \uparrow^{\mathfrak{S}_{a p^{k}}}\left(g^{\prime}\right)$ for $g^{\prime} \in \mathfrak{S}_{a p^{k}}$ of cycle type $p^{l}$, we have by Lemma 4.4 and Theorem 4.13 that

$$
\sum_{i=1}^{a} C_{l}\left(\mathrm{~s}^{i}\right)=\sum_{i=1}^{a} C_{l}\left(\mathrm{t}^{i}\right)
$$

Substituting this into (15) along with the fact that $\phi(\underline{\mathbf{s}}) \uparrow^{\mathfrak{S}_{a p^{k}}}(g)=\phi(\underline{\mathbf{t}}) \uparrow^{\mathfrak{S}_{a p^{k}}}(g)$ for $g \in \mathfrak{S}_{a p^{k}}$ of cycle type $p^{l} p^{l}$, we obtain

By Lemma 4.4, this gives

$$
\sum_{i=1}^{a} C_{l}\left(\mathrm{~s}^{i}\right)^{2}=\sum_{i=1}^{a} C_{l}\left(\mathrm{t}^{i}\right)^{2}
$$

$$
\sum_{i=1}^{a} \prod_{m=1}^{l}\left(p \mathbf{s}_{m}^{i}-1\right)^{2}=\sum_{i=1}^{a} \prod_{m=1}^{l}\left(p \mathrm{t}_{m}^{i}-1\right)^{2}
$$

In other words, we have

$$
\sum_{i=1}^{a}(-q)^{\sigma_{i}}=\sum_{i=1}^{a}(-q)^{\tau_{i}} \quad \text { where } \quad q=p-1, \quad \sigma_{i}=2 \sum_{m=1}^{l} \mathrm{~s}_{m}^{i} \quad \text { and } \quad \tau_{i}=2 \sum_{m=1}^{l} \mathrm{t}_{m}^{i} .
$$

Therefore by Lemma 5.2 we must have $\left\{\sigma_{1}, \ldots, \sigma_{a}\right\}^{*}=\left\{\tau_{1}, \ldots, \tau_{a}\right\}^{*}$ (case (ii) of Lemma 5.2 is not possible as $\sigma_{i}$ and $\tau_{i}$ are even for all $i$ ). The assertion of the present lemma for $l<k$ then follows directly.

Finally, suppose $l=k$. The assertion in this case follows by a similar argument to the case of $l<k$, the only difference being that we have the following instead of (15) because $X(k, k)=\emptyset$ :

$$
\begin{aligned}
\sum_{x \in W(k, k)} \phi(\underline{\mathbf{u}})(x) & =\sum_{\{i, j\} \subseteq[a]} \sum_{x_{i}, x_{j} \in X(k)} \phi\left(\mathbf{u}^{i}\right)\left(x_{i}\right) \cdot \phi\left(\mathbf{u}^{j}\right)\left(x_{j}\right)=\sum_{1 \leq i<j \leq a} \Gamma_{k ; k}\left(\mathbf{u}^{i}\right) \cdot \Gamma_{k ; k}\left(\mathbf{u}^{j}\right) \\
& =\frac{p^{2 k}}{2}\left(\sum_{i=1}^{a} C_{k}\left(\mathbf{u}^{i}\right)\right)\left(\sum_{j=1}^{a} C_{k}\left(\mathbf{u}^{j}\right)\right)-\frac{p^{2 k}}{2} \cdot \sum_{i=1}^{a} C_{k}\left(\mathbf{u}^{i}\right)^{2} .
\end{aligned}
$$

We are now ready to translate the equations from Corollary $[5.1$ into statements concerning multisets of (sums of) partial sums of the sequences $s^{1}, \ldots, s^{a}$ and $t^{1}, \ldots, t^{a}$, as promised.

Proposition 5.4. Let $a \in[p-1]$. Let $\phi(\underline{\mathbf{s}}), \phi(\underline{\mathbf{t}}) \in \operatorname{Lin}\left(P_{a p^{k}}\right)$ and suppose $\phi(\underline{\mathbf{s}}) \uparrow^{\mathfrak{S}_{a p^{k}}}=$ $\phi(\underline{\mathbf{t}})^{\mathfrak{S}_{a p^{k}}}$. Let $b \in[a]$ and let $l_{1}, \ldots, l_{b}$ be distinct integers in $[k]$. Then $\left\{\sigma_{1}, \ldots, \sigma_{a}\right\}^{*}=$ $\left\{\tau_{1}, \ldots, \tau_{a}\right\}^{*}$ where

for each $j \in[a]$.

$$
\sigma_{j}=\sum_{i=1}^{b} \sum_{m=1}^{l_{i}} \mathrm{~s}_{m}^{j} \quad \text { and } \quad \tau_{j}=\sum_{i=1}^{b} \sum_{m=1}^{l_{i}} \mathrm{t}_{m}^{j}
$$

Proof. By Corollary 5.1 and Definition 4.3,

$$
\sum_{j=1}^{a} \prod_{i=1}^{b} \prod_{m=1}^{l_{i}}\left(p \mathbf{s}_{m}^{j}-1\right)=\sum_{j=1}^{a} \prod_{i=1}^{b} \prod_{m=1}^{l_{i}}\left(p \mathbf{t}_{m}^{j}-1\right),
$$


and hence $\sum_{j=1}^{a}(-p+1)^{\sigma_{j}}=\sum_{j=1}^{a}(-p+1)^{\tau_{j}}$. The assertion follows immediately if $a=1$, and if $a>1$ (in which case $p$ is therefore necessarily odd) then it follows from Lemma 5.2 case (ii) of Lemma 5.2 cannot occur because $\sum_{j=1}^{a} \sigma_{j}=\sum_{j=1}^{a} \tau_{j}$ by Lemma 5.3 .

While the conclusion of Theorem 3.6 does not follow instantly from Proposition 5.4, only two more combinatorial arguments are needed to deduce that $\underline{\mathbf{s}}$ is a permutation of $\underline{\mathbf{t}}$. Before this, we give a definition which will simplify the notation and an example to illustrate this.

Definition 5.5. Let $b, k \in \mathbb{N}$. Given natural numbers $l_{1}, \ldots, l_{b} \leq k$ and a sequence $\mathbf{s}=$ $\left(\mathrm{s}_{1}, \ldots, \mathrm{s}_{k}\right) \in\{0,1\}^{k}$, define

$$
f\left(l_{1}, \ldots, l_{b} ; \mathrm{s}\right)=\sum_{i=1}^{b} \sum_{m=1}^{l_{i}} \mathrm{~s}_{m} .
$$

Let $a \in \mathbb{N}$. Given an a-tuple $\underline{\mathbf{s}}=\left(\mathrm{s}^{1}, \ldots, \mathrm{s}^{a}\right)$ where $\mathrm{s}^{i} \in\{0,1\}^{k}$ for all $i$, define $f\left(l_{1}, \ldots, l_{b} ; \underline{\mathbf{s}}\right)$ to be the multiset

$$
f\left(l_{1}, \ldots, l_{b} ; \underline{\mathbf{s}}\right)=\left\{f\left(l_{1}, \ldots, l_{b} ; \mathbf{s}^{1}\right), \ldots, f\left(l_{1}, \ldots, l_{b} ; \mathbf{s}^{a}\right)\right\}^{*} .
$$

Thus the result of Proposition 5.4 may be restated as

$$
f\left(l_{1}, \ldots, l_{b} ; \underline{\mathbf{s}}\right)=f\left(l_{1}, \ldots, l_{b} ; \underline{\mathbf{t}}\right) .
$$

Example 5.6. Let $k=5$ and $a=3$. Let $b=3$ with $l_{1}=2, l_{2}=4$ and $l_{3}=5$. Then

$$
\begin{aligned}
& \mathrm{s}^{1}=(0,0,0,0,1) \\
& \mathrm{s}^{2}=(1,0,1,1,0) \\
& \mathrm{s}^{3}=(1,1,0,0,1)
\end{aligned} \quad \Longrightarrow \quad \begin{aligned}
& f\left(2,4,5 ; \mathrm{s}^{1}\right)=0+0+1=1 \\
& f\left(2,4,5 ; \mathrm{s}^{2}\right)=1+3+3=7 \\
& f\left(2,4,5 ; \mathrm{s}^{3}\right)=2+2+3=7
\end{aligned}
$$

and so $f(2,4,5 ; \underline{\mathbf{s}})=\{1,7,7\}^{*}$.

The following lemma shows that equality of two multisets is preserved when we add 1 to the same number of elements in each multiset.

Lemma 5.7. Let $\left\{l_{1}, \ldots, l_{b}\right\}^{*}=\left\{m_{1}, \ldots, m_{b}\right\}^{*}$. Suppose that in addition we have

$$
\left\{l_{1}+1, \ldots, l_{c}+1, l_{c+1}, \ldots, l_{b}\right\}^{*}=\left\{m_{1}+1, \ldots, m_{c}+1, m_{c+1}, \ldots, m_{b}\right\}^{*}
$$

for some $c \in[b-1]$. Then $\left\{l_{1}, \ldots, l_{c}\right\}^{*}=\left\{m_{1}, \ldots, m_{c}\right\}^{*}$.

Proof. Suppose for the sake of contradiction that $\left\{l_{1}, \ldots, l_{c}\right\}^{*} \neq\left\{m_{1}, \ldots, m_{c}\right\}^{*}$. Let us suppose without loss of generality that $l_{1} \leq \cdots \leq l_{c}$ and $m_{1} \leq \cdots \leq m_{c}$. Let $j \leq c$ be maximal such that $l_{j} \neq m_{j}$, and without loss of generality we assume that $l_{j}<m_{j}$.

Given a multiset $S$ and $v \in \mathbb{N}$ we let $S_{\geq v}=\{x: x \in S, x \geq v\}^{*}$. Observe that since $\left\{l_{1}, \ldots, l_{b}\right\}^{*}=\left\{m_{1}, \ldots, m_{b}\right\}^{*}$ we have that $\left\{l_{1}, \ldots, l_{b}\right\}_{\geq v}^{*}=\left\{m_{1}, \ldots, m_{b}\right\}_{\geq v}^{*}$ for any $v$. Similarly

$$
\left\{l_{1}+1, \ldots, l_{c}+1, l_{c+1}, \ldots, l_{b}\right\}_{\geq v}^{*}=\left\{m_{1}+1, \ldots, m_{c}+1, m_{c+1}, \ldots, m_{b}\right\}_{\geq v}^{*}
$$

for any $v$. We now consider $v=m_{j}+1$. Note that

$$
\begin{aligned}
& \left|\left\{l_{1}+1, \ldots, l_{c}+1, l_{c+1}, \ldots, l_{b}\right\}_{\geq m_{j}+1}^{*}\right|-\left|\left\{l_{1}, \ldots, l_{b}\right\}_{\geq m_{j}+1}^{*}\right| \\
& \quad=\left|\left\{l_{j+1}+1, \ldots, l_{c}+1\right\}_{\geq m_{j}+1}^{*}\right|-\left|\left\{l_{j+1}, \ldots, l_{c}\right\}_{\geq m_{j}+1}^{*}\right|
\end{aligned}
$$

by cancelling off equal elements in the two multisets and noting that $l_{i}<m_{j}$ for all $i \leq j$. Moreover,

$$
\begin{aligned}
& \left|\left\{m_{1}+1, \ldots, m_{c}+1, m_{c+1}, \ldots, m_{b}\right\}_{\geq m_{j}+1}^{*}\right|-\left|\left\{m_{1}, \ldots, m_{b}\right\}_{\geq m_{j}+1}^{*}\right| \\
\geq & \left|\left\{m_{j}+1, \ldots, m_{c}+1\right\}_{\geq m_{j}+1}^{*}\right|-\left|\left\{m_{j}, \ldots, m_{c}\right\}_{\geq m_{j}+1}^{*}\right| \\
= & 1+\left|\left\{m_{j+1}+1, \ldots, m_{c}+1\right\}_{\geq m_{j}+1}^{*}\right|-\left|\left\{m_{j+1}, \ldots, m_{c}\right\}_{\geq m_{j}+1}^{*}\right|
\end{aligned}
$$




$$
=1+\left|\left\{l_{j+1}+1, \ldots, l_{c}+1\right\}_{\geq m_{j}+1}^{*}\right|-\left|\left\{l_{j+1}, \ldots, l_{c}\right\}_{\geq m_{j}+1}^{*}\right|
$$

by maximality of $j$. In particular, we have

$$
\begin{gathered}
\left|\left\{m_{1}+1, \ldots, m_{c}+1, m_{c+1}, \ldots, m_{b}\right\}_{\geq m_{j}+1}^{*}\right|-\left|\left\{m_{1}, \ldots, m_{b}\right\}_{\geq m_{j}+1}^{*}\right| \\
\geq 1+\left|\left\{l_{1}+1, \ldots, l_{c}+1, l_{c+1}, \ldots, l_{b}\right\}_{\geq m_{j}+1}^{*}\right|-\left|\left\{l_{1}, \ldots, l_{b}\right\}_{\geq m_{j}+1}^{*}\right|
\end{gathered}
$$

which is a contradiction.

We are finally ready to deduce Theorem 3.6 from the equalities of the functions $f$ given by Proposition 5.4 and Definition 5.5 .

Theorem 5.8. Let $a, k \in \mathbb{N}$. Let $\underline{\mathbf{s}}=\left(\mathrm{s}^{1}, \ldots, \mathrm{s}^{a}\right)$ and $\underline{\mathbf{t}}=\left(\mathrm{t}^{1}, \ldots, \mathrm{t}^{a}\right)$ where $\mathrm{s}^{i}, \mathrm{t}^{i} \in\{0,1\}^{k}$ for all $i$. Suppose that for any distinct integers $l_{1}, l_{2}, \ldots, l_{b} \in[k]$ such that $b \in[a]$ we have

$$
f\left(l_{1}, \ldots, l_{b} ; \underline{\mathbf{s}}\right)=f\left(l_{1}, \ldots, l_{b} ; \underline{\mathbf{t}}\right) .
$$

Then there exists a permutation $\sigma \in \mathfrak{S}_{a}$ such that $\mathrm{s}^{i}=\mathrm{t}^{\sigma(i)}$ for all $i$.

Proof. We prove the assertion for $(a, k)$ by induction on $a+k$. When $k=1$ and $a$ is arbitrary, the assertion is clear since the single term of each sequence $s^{i}$ is simply $f\left(1 ; s^{i}\right)$. When $a=1$ and $k$ is arbitrary, note that $\mathrm{s}_{1}^{1}=f\left(1 ; \mathrm{s}^{1}\right)=f\left(1 ; \mathrm{t}^{1}\right)=\mathrm{t}_{1}^{1}$, and $\mathrm{s}_{r}^{1}=f\left(r ; \mathrm{s}^{1}\right)-f\left(r-1 ; \mathrm{s}^{1}\right)=$ $f\left(r ; \mathrm{t}^{1}\right)-f\left(r-1 ; \mathrm{t}^{1}\right)=\mathrm{t}_{r}^{1}$ for all $r \in\{2,3, \ldots, k\}$, so $\mathrm{s}^{1}=\mathrm{t}^{1}$ as required.

Now suppose $a, k \geq 2$. Write $\hat{\mathbf{s}}^{i}$ for the sequence $\left(\mathrm{s}_{j}^{i}\right)_{j=2}^{k} \in\{0,1\}^{k-1}$ and let $\underline{\hat{\mathbf{s}}}=\left(\hat{\mathbf{s}}^{1}, \ldots, \hat{\mathrm{s}}^{a}\right)$. Define $\hat{\mathrm{t}}^{i}$ and $\underline{\hat{\mathrm{t}}}$ similarly.

First suppose that $\mathrm{s}_{1}^{i}=\mathrm{t}_{1}^{i}=z$ for all $i \in[a]$, for some $z \in\{0,1\}$. Observe that for any distinct integers $l_{1}, \ldots, l_{b} \in[k-1]$ such that $b \in[a]$ we have

$$
f\left(l_{1}+1, \ldots, l_{b}+1 ; s^{i}\right)=f\left(l_{1}, \ldots, l_{b} ; \hat{\mathrm{s}}^{i}\right)+b z,
$$

and similarly

$$
f\left(l_{1}+1, \ldots, l_{b}+1 ; \mathrm{t}^{i}\right)=f\left(l_{1}, \ldots, l_{b} ; \hat{\mathrm{t}}^{i}\right)+b z .
$$

Thus by (16), we have that

$$
f\left(l_{1}, \ldots, l_{b} ; \underline{\hat{\mathbf{s}}}\right)=f\left(l_{1}, \ldots, l_{b} ; \underline{\hat{\mathrm{t}}}\right) .
$$

By the inductive hypothesis for $(a, k-1)$, there exists a permutation $\sigma \in \operatorname{Sym}[a]$ such that $\hat{\mathrm{s}}^{i}=\hat{\mathrm{t}}^{\sigma(i)}$ for all $i \in[a]$. Therefore $\mathrm{s}^{i}=\mathrm{t}^{\sigma(i)}$ as required.

Otherwise, we may now suppose that not all $\mathrm{s}_{1}^{i}$ and $\mathrm{t}_{1}^{i}$ are equal. Let $I_{s}=\left\{i \in[a]: \mathrm{s}_{1}^{i}=1\right\}$ and define $I_{t}$ similarly. Since $f(1 ; \underline{\mathbf{s}})=f(1 ; \underline{\mathbf{t}})$, then $\left|I_{s}\right|=\left|I_{t}\right|$, so we may without loss of generality reorder $\underline{\mathbf{t}}$ to assume that $\mathrm{s}_{1}^{i}=\mathrm{t}_{1}^{i}$ for all $i \in[a]$. Let $I=I_{s}=I_{t}$ and note that $|I| \in[a-1]$.

For any distinct integers $l_{1}, \ldots, l_{b} \in[k-1]$ such that $b \in[a-1]$ we have

$$
f\left(l_{1}+1, \ldots, l_{b}+1 ; s^{i}\right)=b s_{1}^{i}+f\left(l_{1}, \ldots, l_{b} ; \hat{s}^{i}\right)
$$

and

since $\mathrm{s}_{1}^{i}=\mathrm{t}_{1}^{i}$. Thus

$$
f\left(l_{1}+1, \ldots, l_{b}+1 ; \mathrm{t}^{i}\right)=b \mathrm{~s}_{1}^{i}+f\left(l_{1}, \ldots, l_{b} ; \hat{\mathrm{t}}^{i}\right)
$$

$$
\begin{aligned}
f\left(l_{1}+1, \ldots, l_{b}+1 ; \underline{\mathbf{s}}\right)=\left\{b s_{1}^{i}+f\left(l_{1}, \ldots, l_{b} ; \hat{\mathbf{s}}^{i}\right): i \in[a]\right\}^{*} \\
\quad=f\left(l_{1}+1, \ldots, l_{b}+1 ; \underline{\mathbf{t}}\right)=\left\{b s_{1}^{i}+f\left(l_{1}, \ldots, l_{b} ; \hat{\mathrm{t}}^{i}\right): i \in[a]\right\}^{*} .
\end{aligned}
$$

In addition,

$$
\begin{aligned}
& f\left(1, l_{1}+1, \ldots, l_{b}+1 ; \underline{\mathbf{s}}\right)=\left\{(b+1) \mathbf{s}_{1}^{i}+f\left(l_{1}, \ldots, l_{b} ; \hat{\mathrm{s}}^{i}\right): i \in[a]\right\}^{*} \\
& \quad=f\left(1, l_{1}+1, \ldots, l_{b}+1 ; \underline{\mathbf{t}}\right)=\left\{(b+1) \mathbf{s}_{1}^{i}+f\left(l_{1}, \ldots, l_{b} ; \hat{\mathrm{t}}^{i}\right): i \in[a]\right\}^{*} .
\end{aligned}
$$


Therefore, by Lemma 5.7, we have that

$$
\left\{b+f\left(l_{1}, \ldots, l_{b} ; \hat{\mathrm{s}}^{i}\right): i \in I\right\}^{*}=\left\{b+f\left(l_{1}, \ldots, l_{b} ; \hat{\mathrm{t}}^{i}\right): i \in I\right\}^{*},
$$

which implies

$$
\left\{f\left(l_{1}, \ldots, l_{b} ; \hat{\mathrm{s}}^{i}\right): i \in I\right\}^{*}=\left\{f\left(l_{1}, \ldots, l_{b} ; \hat{\mathrm{t}}^{i}\right): i \in I\right\}^{*}
$$

and therefore by (17) also

$$
\left\{f\left(l_{1}, \ldots, l_{b} ; \hat{\mathrm{s}}^{i}\right): i \in[a] \backslash I\right\}^{*}=\left\{f\left(l_{1}, \ldots, l_{b} ; \hat{\mathrm{t}}^{i}\right): i \in[a] \backslash I\right\}^{*} .
$$

Let $\underline{\hat{\mathbf{s}}}^{(1)}$ and $\underline{\hat{\mathbf{s}}}^{(0)}$ be the sequences $\left(\hat{\mathbf{s}}^{i}\right)_{i \in I}$ and $\left(\hat{\mathrm{s}}^{i}\right)_{i \notin I}$ respectively, and define $\underline{\hat{\mathbf{t}}}^{(1)}$ and $\underline{\hat{\mathbf{t}}}^{(0)}$ similarly. Since (18) and (19) hold for any valid choice of $\left\{l_{i}\right\}$, this tells us that for any distinct integers $l_{1}, \ldots, l_{b} \in[k-1]$ such that $b \in[a-1]$ we have

$$
f\left(l_{1}, \ldots, l_{b} ; \underline{\hat{\mathbf{s}}}^{(1)}\right)=f\left(l_{1}, \ldots, l_{b} ; \underline{\hat{\mathbf{t}}}^{(1)}\right) \quad \text { and } \quad f\left(l_{1}, \ldots, l_{b} ; \underline{\hat{\mathbf{s}}}^{(0)}\right)=f\left(l_{1}, \ldots, l_{b} ; \underline{\hat{\mathbf{t}}}^{(0)}\right) .
$$

Since $|I|$ and $a-|I|$ are both at most $a-1$, we may apply the inductive hypotheses for $(|I|, k-1)$ and $(a-|I|, k-1)$ to obtain permutations $\sigma_{1} \in \operatorname{Sym} I$ such that $\hat{\mathrm{s}}^{i}=\hat{\mathrm{t}}^{\sigma_{1}(i)}$ for all $i \in I$ (and hence $\left.\mathrm{s}^{i}=\mathrm{t}^{\sigma_{1}(i)}\right)$ and $\sigma_{0} \in \operatorname{Sym}([a] \backslash I)$ such that $\hat{\mathrm{s}}^{i}=\hat{\mathrm{t}}^{\sigma_{0}(i)}$ for all $i \notin I$ (and hence $\mathrm{s}^{i}=\mathrm{t}^{\sigma_{0}(i)}$ ). Finally, let $\sigma=\sigma_{0} \cdot \sigma_{1} \in \operatorname{Sym}[a]$, so $\mathrm{s}^{i}=\mathrm{t}^{\sigma(i)}$ for all $i$ as desired.

Proof of Theorem 3.6. This follows from Proposition 5.4 and Theorem 5.8

Proof of Theorem 2.2. This is equivalent to Theorem 3.6.

\section{Appendix A. Examples for Proposition 4.9}

We follow the notation in the statement of Proposition 4.9.

Example A.1. In this example, we prove that Proposition 4.9 holds when $b=2$, i.e. that

$$
\Gamma_{l_{1}, l_{2} ; k}(\mathbf{u})=p^{k} \cdot C_{l_{1}}(\mathbf{u}) \cdot C_{l_{2}}(\mathbf{u}) \cdot\left(p^{k}-p^{l_{2}}\right) .
$$

First, we have by combining Definitions 4.2 and 4.3 that

$$
\Gamma_{l_{1}, l_{2} ; k}(\mathrm{u})=\sum_{x \in X\left(l_{1}, l_{2}\right)} \phi(\mathbf{u})(x) .
$$

Consider some $x \in X\left(l_{1}, l_{2}\right)$. Since $l_{1}<l_{2}<k$, then $x$ must have a fixed point. Therefore $x=\left(f_{1}, \ldots, f_{p} ; 1\right)$ with $f_{1}, \ldots, f_{p} \in P_{p^{k-1}}$, and

(i) either $f_{i} \in Y\left(l_{1}\right), f_{j} \in Y\left(l_{2}\right)$ for some distinct $i, j \in[p]$ and $f_{h}=1$ for all $h \notin\{i, j\}$,

(ii) or $f_{i} \in Y\left(l_{1}, l_{2}\right)$ for a unique $i \in[p]$ and $f_{j}=1$ for all $j \neq i$.

By Lemma 3.1, in case (i) we have $\phi(\mathbf{u})(x)=\phi\left(\mathbf{u}^{-}\right)\left(f_{i}\right) \cdot \phi\left(\mathbf{u}^{-}\right)\left(f_{j}\right)$, while in case (ii) we have $\phi(\mathrm{u})(x)=\phi\left(\mathbf{u}^{-}\right)\left(f_{i}\right)$. Since there are $p(p-1)$ choices for the ordered pair $(i, j)$ in case (i) and $p$ choices for $i$ in case (ii),

$$
\Gamma_{l_{1}, l_{2} ; k}(\mathbf{u})=p(p-1) \cdot \Gamma_{l_{1} ; k-1}\left(\mathbf{u}^{-}\right) \cdot \Gamma_{l_{2} ; k-1}\left(\mathbf{u}^{-}\right)+p \cdot \Gamma_{l_{1}, l_{2} ; k-1}\left(\mathbf{u}^{-}\right) .
$$

Iterating (A.1(b) and recalling that $\Gamma_{l_{1}, l_{2} ; l_{2}}(-)=0$, we therefore have that

$$
\Gamma_{l_{1}, l_{2} ; k}(\mathbf{u})=\sum_{i=l_{2}}^{k-1} p^{k-i}(p-1) \cdot \Gamma_{l_{1} ; i}\left(\left(\mathbf{u}_{1}, \ldots, \mathbf{u}_{i}\right)\right) \cdot \Gamma_{l_{2} ; i}\left(\left(\mathbf{u}_{1}, \ldots, \mathbf{u}_{i}\right)\right),
$$


From Lemma 4.4, we know that $\Gamma_{l_{j} ; i}\left(\left(\mathbf{u}_{1}, \ldots, \mathbf{u}_{i}\right)\right)=p^{i} C_{l_{j}}(\mathbf{u})$ for any $j$, and so

$$
\Gamma_{l_{1}, l_{2} ; k}(\mathbf{u})=\sum_{i=l_{2}}^{k-1} p^{k-i}(p-1) \cdot p^{i} C_{l_{1}}(\mathbf{u}) \cdot p^{i} C_{l_{2}}(\mathbf{u})
$$

Define $\Sigma_{2}$ by setting $\Gamma_{l_{1}, l_{2} ; k}(\mathrm{u})=\Sigma_{2} \cdot p^{k} C_{l_{1}}(\mathrm{u}) C_{l_{2}}(\mathrm{u})$, i.e.

$$
\Sigma_{2}:=\sum_{i=l_{2}}^{k-1} p^{-i}(p-1) \cdot p^{i} \cdot p^{i}
$$

Since

$$
\Sigma_{2}=\sum_{i=l_{2}}^{k-1}(p-1) p^{i}=p^{k}-p^{l_{2}}
$$

we obtain

$$
\Gamma_{l_{1}, l_{2} ; k}(\mathbf{u})=\sum_{i=l_{2}}^{k-1} p^{k-i}(p-1) \cdot p^{i} C_{l_{1}}(\mathbf{u}) \cdot p^{i} C_{l_{2}}(\mathbf{u})=p^{k} \cdot C_{l_{1}}(\mathbf{u}) \cdot C_{l_{2}}(\mathbf{u}) \cdot\left(p^{k}-p^{l_{2}}\right) .
$$

This concludes the case of $b=2$.

Example A.2. We prove that Proposition 4.9 holds when $b=3$, i.e. that

$$
\Gamma_{l_{1}, l_{2}, l_{3} ; k}(\mathrm{u})=p^{k} \cdot C_{l_{1}}(\mathrm{u}) \cdot C_{l_{2}}(\mathrm{u}) \cdot C_{l_{3}}(\mathrm{u}) \cdot\left(p^{k}-p^{l_{3}}\right)\left(p^{k}-p^{l_{3}}-p^{l_{2}}\right),
$$

using the case of $b=2$. We thereby illustrate a specific example of the inductive step in the proof of Proposition 4.9, In the calculations below, we will associate certain terms with partitions $\nu$ of the set $[b]=\{1,2,3\}$. This is to help illustrate the analogy between the case of $b=3$ and the general proof for all $b \geq 3$ in Section 4. Moreover, equations (A.2(a)-(f)) below are labelled as such to illustrate their correspondence to equations (A.1(a)-(f)) above.

From Definitions 4.2 and 4.3 , we have

$$
\Gamma_{l_{1}, l_{2}, l_{3} ; k}(\mathbf{u})=\sum_{x \in X\left(l_{1}, l_{2}, l_{3}\right)} \phi(\mathbf{u})(x) .
$$

Consider some $x \in X\left(l_{1}, l_{2}, l_{3}\right)$. Since $x$ has a fixed point, $x$ must be of the form $x=\left(f_{1}, \ldots, f_{p} ; 1\right)$ with $f_{1}, \ldots, f_{p} \in P_{p^{k-1}}$, and so

$$
\phi(\mathbf{u})(x)=\phi\left(\mathbf{u}^{-}\right)\left(f_{1}\right) \cdot \phi\left(\mathbf{u}^{-}\right)\left(f_{2}\right) \cdots \phi\left(\mathbf{u}^{-}\right)\left(f_{p}\right)
$$

by Lemma 3.1. Moreover, the product of the cycle types of $f_{i}$ over all $i$ equals $p^{l_{1}} p^{l_{2}} p^{l_{3}}$ since $x \in X\left(l_{1}, l_{2}, l_{3}\right)$. We can thus associate to $x$ a partition $\nu$ of the set $\{1,2,3\}$ according to which $p^{l_{w}}(w \in\{1,2,3\})$ occur in the cycle type of $f_{1}, \ldots, f_{p}$ as follows.

For each $i$, say $f_{i} \in Y\left(\left\{l_{w}: w \in \nu_{i}\right\}\right)$ for some subset $\nu_{i} \subseteq\{1,2,3\}$, and $\nu_{1}, \ldots, \nu_{p}$ are disjoint with $\nu_{1} \cup \cdots \cup \nu_{p}=\{1,2,3\}$. (If $\nu_{i}=\emptyset$ then $f_{i}=1$.) In other words, $\nu=\left\{\nu_{i}: \nu_{i} \neq \emptyset\right\} \in$ Part $\{1,2,3\}$ describes how the cycle lengths $p^{l_{w}}$ are grouped together to give the cycle types of $f_{1}, \ldots, f_{p}$. Given $\nu$, there are $p(p-1) \cdots(p-|\nu|+1)$ many ways of assigning the elements of $\nu$ to $f_{1}, \ldots, f_{p}$. 
Hence, by grouping $x \in X\left(l_{1}, l_{2}, l_{3}\right)$ according to its associated $\nu \in \operatorname{Part}\{1,2,3\}$, A.2(a) may be rewritten as follows:

$$
\begin{array}{rlrl}
\Gamma_{l_{1}, l_{2}, l_{3} ; k}(\mathrm{u})= & p \cdot \Gamma_{l_{1}, l_{2}, l_{3} ; k-1}\left(\mathrm{u}^{-}\right) & & \nu=\{\{1,2,3\}\} \\
& +p(p-1) \cdot \Gamma_{l_{1}, l_{2} ; k-1}\left(\mathrm{u}^{-}\right) \cdot \Gamma_{l_{3} ; k-1}\left(\mathbf{u}^{-}\right) & & \nu=\{\{1,2\},\{3\}\} \\
& +p(p-1) \cdot \Gamma_{l_{1}, l_{3} ; k-1}\left(\mathbf{u}^{-}\right) \cdot \Gamma_{l_{2} ; k-1}\left(\mathbf{u}^{-}\right) & \nu=\{\{1,3\},\{2\}\} \\
& +p(p-1) \cdot \Gamma_{l_{2}, l_{3} ; k-1}\left(\mathbf{u}^{-}\right) \cdot \Gamma_{l_{1} ; k-1}\left(\mathbf{u}^{-}\right) & \nu=\{\{2,3\},\{1\}\} \\
& +p(p-1)(p-2) \cdot \Gamma_{l_{1} ; k-1}\left(\mathbf{u}^{-}\right) \cdot \Gamma_{l_{2} ; k-1}\left(\mathbf{u}^{-}\right) \cdot \Gamma_{l_{3} ; k-1}\left(\mathbf{u}^{-}\right) & & \nu=\{\{1\},\{2\},\{3\}\}
\end{array}
$$
that

Let $\mathrm{v}_{i}=\left(\mathrm{u}_{1}, \ldots, \mathrm{u}_{i}\right)$. Iterating (A.2(b) and recalling that $\Gamma_{l_{1}, l_{2}, l_{3} ; l_{3}}(-)=0$, we therefore have

$$
\begin{aligned}
\Gamma_{l_{1}, l_{2}, l_{3} ; k}(\mathrm{u})=\sum_{i=l_{3}}^{k-1} p^{k-i}[ & (p-1) \cdot \Gamma_{l_{1}, l_{2} ; i}\left(\mathrm{v}_{i}\right) \cdot \Gamma_{l_{3} ; i}\left(\mathrm{v}_{i}\right) & \nu & =\{\{1,2\},\{3\}\} \\
& +(p-1) \cdot \Gamma_{l_{1}, l_{3} ; i}\left(\mathrm{v}_{i}\right) \cdot \Gamma_{l_{2} ; i}\left(\mathrm{v}_{i}\right) & \nu & =\{\{1,3\},\{2\}\} \\
& +(p-1) \cdot \Gamma_{l_{2}, l_{3} ; i}\left(\mathrm{v}_{i}\right) \cdot \Gamma_{l_{1} ; i}\left(\mathrm{v}_{i}\right) & \nu & =\{\{2,3\},\{1\}\} \\
& \left.+(p-1)(p-2) \cdot \Gamma_{l_{1} ; i}\left(\mathrm{v}_{i}\right) \cdot \Gamma_{l_{2} ; i}\left(\mathrm{v}_{i}\right) \cdot \Gamma_{l_{3} ; i}\left(\mathrm{v}_{i}\right)\right] & \nu & =\{\{1\},\{2\},\{3\}\}
\end{aligned}
$$

We use Lemma 4.4 and the $b=2$ case of Proposition 4.9 to deduce that

$$
\begin{array}{rlrl}
\Gamma_{l_{1}, l_{2}, l_{3} ; k}(\mathrm{u})=\sum_{i=l_{3}}^{k-1} p^{k-i}[ & (p-1) \cdot p^{i} C_{l_{1}}(\mathrm{u}) C_{l_{2}}(\mathrm{u})\left(p^{i}-p^{l_{2}}\right) \cdot p^{i} C_{l_{3}}(\mathrm{u}) \\
& +(p-1) \cdot p^{i} C_{l_{1}}(\mathrm{u}) C_{l_{3}}(\mathrm{u})\left(p^{i}-p^{l_{3}}\right) \cdot p^{i} C_{l_{2}}(\mathrm{u}) \\
& +(p-1) \cdot p^{i} C_{l_{2}}(\mathrm{u}) C_{l_{3}}(\mathrm{u})\left(p^{i}-p^{l_{3}}\right) \cdot p^{i} C_{l_{1}}(\mathrm{u}) \\
& \left.+(p-1)(p-2) \cdot p^{i} C_{l_{1}}(\mathrm{u}) \cdot p^{i} C_{l_{2}}(\mathrm{u}) \cdot p^{i} C_{l_{3}}(\mathrm{u})\right] \\
=\sum_{i=l_{3}}^{k-1} p^{k-i} \cdot C_{l_{1}}(\mathrm{u}) C_{l_{2}}(\mathrm{u}) C_{l_{3}}(\mathrm{u})[ & (p-1) \cdot p^{2 i}\left(p^{i}-p^{l_{2}}\right) & \nu=\{\{1,2\},\{3\}\} \\
& +(p-1) \cdot p^{2 i}\left(p^{i}-p^{l_{3}}\right) & \nu=\{\{1,3\},\{2\}\} \\
& +(p-1) \cdot p^{2 i}\left(p^{i}-p^{l_{3}}\right) & \nu=\{\{2,3\},\{1\}\} \\
& \left.+(p-1)(p-2) \cdot p^{3 i}\right] \quad \nu=\{\{1\},\{2\},\{3\}\}
\end{array}
$$

Thus, it remains to prove that $\Sigma_{3}=\left(p^{k}-p^{l_{3}}\right)\left(p^{k}-p^{l_{3}}-p^{l_{2}}\right)$, where we define $\Sigma_{3}$ as $\Sigma_{3}:=\sum_{i=l_{3}}^{k-1} p^{-i}\left[(p-1) p^{2 i}\left(p^{i}-p^{l_{2}}\right)+(p-1) p^{2 i}\left(p^{i}-p^{l_{3}}\right)+(p-1) p^{2 i}\left(p^{i}-p^{l_{3}}\right)+(p-1)(p-2) p^{3 i}\right]$

Let $Q_{i}=(p-1) p^{i}$, and recall that $\sum_{i=l_{3}}^{k-1} Q_{i}=p^{k}-p^{l_{3}}$ (see (A.1) from the case of $\left.b=2\right)$. Note further that

$$
\sum_{j=l_{3}}^{i-1} Q_{j}=p^{i}-p^{l_{3}} \quad \text { for all } i \in \mathbb{N} \text { such that } i>l_{3} .
$$

We group the terms appearing in $\Sigma_{3}$ by their associated $\nu \in$ Part $\{1,2,3\}$ according to the partition $\gamma \in$ Part $\{2,3\}$ obtained from $\nu$ by removing 1 . That is, we group the terms as follows:

(i) $\gamma=\{\{2\},\{3\}\}: \nu=\{\{1,2\},\{3\}\}, \nu=\{\{1,3\},\{2\}\}, \nu=\{\{1\},\{2\},\{3\}\}$, and

(ii) $\gamma=\{\{2,3\}\}: \nu=\{\{2,3\},\{1\}\}$.

By first summing together the terms corresponding to the same $\gamma$, we obtain

$$
\Sigma_{3}=\sum_{i=l_{3}}^{k-1}[\underbrace{(p-1) p^{i}\left(p^{i+1}-p^{l_{3}}-p^{l_{2}}\right)}_{\gamma=\{\{2\},\{3\}\}}+\underbrace{(p-1) p^{i}\left(p^{i}-p^{l_{3}}\right)}_{\gamma=\{\{2,3\}\}}]
$$




$$
\begin{aligned}
& =\sum_{i=l_{3}}^{k-1}\left[Q_{i} \cdot\left(p^{i+1}-p^{l_{3}}-p^{l_{2}}\right)+(p-1) p^{i}\left(p^{i}-p^{l_{3}}\right)\right] \\
& =\left(-p^{l_{3}}-p^{l_{2}}\right) \sum_{i=l_{3}}^{k-1} Q_{i}+\sum_{i=l_{3}}^{k-1}\left[p^{i+1} Q_{i}+(p-1) p^{i}\left(p^{i}-p^{l_{3}}\right)\right] \\
& =\left(-p^{l_{3}}-p^{l_{2}}\right)\left(p^{k}-p^{l_{3}}\right)+\sum_{i=l_{3}}^{k-1}\left[p^{i+1} Q_{i}+(p-1) p^{i} \sum_{j=l_{3}}^{i-1} Q_{j}\right] \\
& =\left(-p^{l_{3}}-p^{l_{2}}\right)\left(p^{k}-p^{l_{3}}\right)+\sum_{h=l_{3}}^{k-2} Q_{h} \cdot\left(p^{h+1}+(p-1) \sum_{z=h+1}^{k-1} p^{z}\right)+p^{k} Q_{k-1} \\
& =\left(-p^{l_{3}}-p^{l_{2}}\right)\left(p^{k}-p^{l_{3}}\right)+p^{k} \sum_{h=l_{3}}^{k-1} Q_{h} \\
& =\left(-p^{l_{3}}-p^{l_{2}}\right)\left(p^{k}-p^{l_{3}}\right)+p^{k}\left(p^{k}-p^{l_{3}}\right)=\left(p^{k}-p^{l_{3}}\right)\left(p^{k}-p^{l_{3}}-p^{l_{2}}\right),
\end{aligned}
$$

as desired.

\section{REFERENCES}

[G21] E. GiannelLi, McKay bijections for symmetric and alternating groups, Algebra Number Theory, to appear.

[GL18] E. Giannelli and S. LaW, On permutation characters and Sylow p-subgroups of $\mathfrak{S}_{n}, J$. Algebra 506 (2018), 409-428.

[GL20] E. Giannelli and S. LaW, Sylow branching coefficients for symmetric groups, J. London Math. Soc., 103 no. 2 (2021), 697-728.

[I76] I. M. IsAACS, Character theory of finite groups, Dover, New York, 1976.

[J78] G. James, The Representation Theory of the Symmetric Groups, Lecture Notes in Mathematics 682, Springer, Berlin, 1978.

[JK81] G. JAMES AND A. KERBER, The representation theory of the symmetric group, Encyclopedia of Mathematics and its Applications, vol. 16, Addison-Wesley Publishing Co., Reading, Mass., 1981.

[L19] S. LAw, On problems in the representation theory of symmetric groups, Ph.D. thesis, University of Cambridge, 2019.

[MN12] G. Malle AND G. NAvarRo, Characterizing normal Sylow p-subgroups by character degrees, J. Algebra 370 (2012) 402-406.

[N03] G. Navarro, Linear characters of Sylow subgroups, J. Algebra 269 (2003), 589-598.

[N18] G. NAvarro, Character tables and Sylow subgroups revisited, in N. S. Narasimha Sastry, M. K. Yadav (eds) (2018), Group theory and computation, Indian Stat. Inst. Ser., Springer, Singapore, 197-206.

[NTV14] G. Navarro, P. H. TieP And C. Vallejo, McKay natural correspondences on characters, Algebra Number Theory 8 no. 8 (2014), 1839-1856. ITALY

(E. Giannelli) Dipartimento di Matematica e Informatica U. Dini, Viale Morgagni 67/a, Firenze,

Email address: eugenio.giannelli@unifi.it

(S. Law) Department of Pure Mathematics and Mathematical Statistics, University of CamBridge, CAmbridge CB3 0WB, UK

Email address: swcl2@cam.ac.uk

(J. Long) Mathematical Institute, University of Oxford, Radcliffe Observatory, Andrew Wiles Building, Woodstock Rd, Oxford OX2 6GG, UK

Email address: jason.long@maths.ox.ac.uk 